The Astrophysical Journal, 675:1141-1155, 2008 March 10

(C) 2008. The American Astronomical Society. All rights reserved. Printed in U.S.A.

\title{
EVOLUTION OF THE BAR FRACTION IN COSMOS: QUANTIFYING THE ASSEMBLY OF THE HUBBLE SEQUENCE
}

\author{
Kartik Sheth, ${ }^{1,2}$ Debra Meloy Elmegreen, ${ }^{3}$ Bruce G. Elmegreen, ${ }^{4}$ Peter Capak, ${ }^{2}$ Roberto G. Abraham, ${ }^{5}$ \\ E. Athanassoula, ${ }^{6}$ Richard S. Ellis, ${ }^{2}$ Bahram Mobasher, ${ }^{7}$ Mara Salvato, ${ }^{2}$ Eva Schinnerer, ${ }^{8}$ \\ Nicholas Z. Scoville, ${ }^{2}$ Lori Spalsbury, ${ }^{2}$ Linda Strubbe, ${ }^{9}$ Marcella Carollo, ${ }^{10}$ \\ Michael Rich, ${ }^{11}$ AND ANDREW A. West 9 \\ Received 2007 July 27; accepted 2007 October 23
}

\begin{abstract}
We have analyzed the redshift-dependent fraction of galactic bars over $0.2<z<0.84$ in 2157 luminous face-on spiral galaxies from the COSMOS $2 \mathrm{deg}^{2}$ field. Our sample is an order of magnitude larger than that used in any previous investigation, and is based on substantially deeper imaging data than that available from earlier wide-area studies of high-redshift galaxy morphology. We find that the fraction of barred spirals declines rapidly with redshift. Whereas in the local universe about $65 \%$ of luminous spiral galaxies contain bars (SB+SAB), at $z \sim 0.84$ this fraction drops to about $20 \%$. Over this redshift range the fraction of strong bars (SBs) drops from about 30\% to under $10 \%$. It is clear that when the universe was half its present age, the census of galaxies on the Hubble sequence was fundamentally different from that of the present day. A major clue to understanding this phenomenon has also emerged from our analysis, which shows that the bar fraction in spiral galaxies is a strong function of stellar mass, integrated color and bulge prominence. The bar fraction in very massive, luminous spirals is about constant out to $z \sim 0.84$, whereas for the low-mass, blue spirals it declines significantly with redshift beyond $z=0.3$. There is also a slight preference for bars in bulge-dominated systems at high redshifts that may be an important clue toward the coevolution of bars, bulges, and black holes. Our results thus have important ramifications for the processes responsible for galactic downsizing, suggesting that massive galaxies matured early in a dynamical sense, and not just as a result of the regulation of their star formation rate.
\end{abstract}

Subject headings: galaxies: evolution — galaxies: general — galaxies: high-redshift — galaxies: spiral — galaxies: structure

\section{INTRODUCTION}

How, when, and at what rate did the Hubble sequence form? This question is central to the field of galaxy formation and evolution. We examine it by measuring the evolution of the bar fraction with redshift using the $2 \mathrm{deg}^{2}$ Cosmic Evolution Survey (COSMOS). In nearly all simulations, the formation timescale for a bar is rapid once the necessary conditions (a massive, dynamically cold, and rotationally supported disk) are met. Therefore, the redshift evolution of the bar fraction is a fundamental probe of the evolutionary history of disk galaxies.

The bar fraction is defined simply as

$$
f_{\text {bar }}=\frac{\text { number of barred spirals }}{\text { number of all spirals }} .
$$

\footnotetext{
1 Spitzer Science Center, California Institute of Technology, Pasadena, CA 91125.

2 California Institute of Technology, MC 105-24, 1200 East California Boulevard, Pasadena, CA 91125.

3 Department of Physics and Astronomy, Vassar College, 124 Raymond Avenue, Poughkeepsie, NY 12604.

4 IBM T. J. Watson Center, P.O. Box 218, Yorktown Heights, NY 10598

5 Department of Astronomy and Astrophysics, University of Toronto, 50 St. George St, Room 1403, Toronto, ON M5S 3H4, Canada.

6 LAM, OAMP, 2 place Le Verrier, 13248 Marseille cedex 04, France.

7 Space Telescope Science Institute, 3700 San Martin Drive, Baltimore, MD 21218.

8 Max Planck Institut für Astronomie, Königstuhl 17, Heidelberg, D-69117, Germany.

9 Astronomy Department, University of California, 601 Campbell Hall, Berkeley, CA 94720-3411.

10 Department of Physics, ETH Zurich, CH-8093 Zurich, Switzerland.

11 Department of Physics and Astronomy, University of California, Los Angeles, CA 90095.
}

In the local universe the value of $f_{\text {bar }}$ is quite well established. When only strongly barred ${ }^{12}$ galaxies (SBs) are counted, the RSA, RC3, and UGC (Sandage \& Tammann 1987; de Vaucouleurs 1991; Nilson 1973) all give values of $f_{\mathrm{bar}}=0.25-0.3$. When ovally distorted (SAB) are also counted the situation becomes a little less clear cut, because, unlike the RC3, the UGC and RSA do not attempt to carefully compile an inventory of such galaxies. If ovally distorted systems in the RC3 are included in the computation of $f_{\text {bar }}$ then the local bar fraction rises to $f_{\text {bar }} \sim$ 0.6 . This result is in good agreement with recent infrared studies, which have measured the local bar fraction to be $\sim 0.65$ (Eskridge et al. 2000; Whyte et al. 2002; Menéndez-Delmestre et al. 2007; Marinova \& Jogee 2007). In the infrared, a majority of the SAB galaxies are classified as strongly barred SB systems (Eskridge et al. 2000). As noted by Eskridge et al. (2000) and Menéndez-Delmestre et al. (2007), the overall bar fraction is the same in the infrared and the optical (although there is a small number of cases where bars are unveiled at infrared wavelengths). This is not surprising, because bars are primarily stellar structures whose visibility only declines sharply at ultraviolet wavelengths, shortward of the Balmer break (see also Appendix A.1). We conclude that the consensus value of the local barred fraction is $f_{\text {bar }} \sim 0.3$ for strongly barred systems, and $f_{\text {bar }} \sim 0.65$ for all barred galaxies, and that these values are so well known that they have not changed significantly in over four decades.

In sharp contrast with the rapid and stable consensus reached on the local bar fraction, attempts to measure the bar fraction at

\footnotetext{
12 Bars that are highly elliptical and have rectangular isophotes are classified as $\mathrm{SB}$ galaxies whereas those with more oval shapes are classified as SAB or ovally distorted galaxies.
} 
high redshift have proven difficult. The earliest analyses of the bar fraction in the Hubble Deep Fields (HDFs) found a dramatic paucity of barred spirals at $z>0.5$ (Abraham et al. 1996, 1999; van den Bergh et al. 1996). These authors concluded that at lookback times greater than 5 Gyr disks were either dark matter dominated or dynamically too hot (perhaps due to the increased merging activity) to host bars. However, the small volume probed by the HDFs (only 30 bright, face-on spiral galaxies between $0<z<1$ ) led to concerns that the bar fraction at high redshift may not be adequately measured. Sheth et al. (2003) investigated whether a significant number of bars could have been missed, as suggested by Bunker (1999) using the $H$-band NICMOS HDF. Sheth et al. (2003) found four bars and two candidate bars out of 95 galaxies at $z>0.7$. Overall, the fraction of barred spirals in the NICMOS HDF remained extremely low, as in the optical HDF studies. But Sheth et al. (2003) noted that their study was limited by the coarse NICMOS resolution $\left(0.15^{\prime \prime}\right)$ such that only the largest (and rarest) bars could be identified (bars with semimajor axis $>5 \mathrm{kpc}$ ). When the fraction of these large bars at $z>0.7$ was compared to local samples, there was no compelling evidence for a decline in barred spirals, but likewise the NICMOS data did not unveil any new bars at low redshifts; all except one of the four bars in the Sheth et al. (2003) study are at $z>0.9$, where $k$-correction effects are important (Appendix A.1).

A major advance in spatial resolution was possible with the Advanced Camera for Surveys (ACS) whose $0.05^{\prime \prime}$ pixels are able to resolve all but the smallest (nuclear, $<2 \mathrm{kpc}$ diameter) bars at all redshifts. Using ACS data, two studies (Elmegreen et al. 2004; Jogee et al. 2004) found that contrary to the previous HDF results, the bar fraction is constant at $30 \%$ over the last 8 Gyr (since $z=1.2$ ). The sample sizes, however, remained modest in these studies (186 in Elmegreen et al. 2004, and 258 in Jogee et al. 2004).

In this paper we examine in detail the redshift evolution of the bar fraction using the unparalleled wide and deep $2 \mathrm{deg}^{2}$ COSMOS data set. The plan for the paper is as follows: in $\S 2$ we describe our sample selection procedure. The classification methodology we have adopted is described in $\S 3$. Our main results are presented in $\S 4$, before being discussed in $\S 5$. Our conclusions are summarized in $\S 6$. Appendix A of this paper provides a detailed analysis of possible selection effects at high redshift and a discussion of our local calibration sample of 139 galaxies from the Sloan Digital Sky Survey (SDSS) Data Release 4 (Adelman-McCarthy et al. 2006). Throughout this paper we adopt a flat $\Lambda$-dominated cosmology with $H_{0}=$ $70 \mathrm{~km} \mathrm{~s}^{-1} \mathrm{Mpc}^{-1}, \Omega_{M}=0.3$, and $\Omega_{\Lambda}=0.7$.

\section{SAMPLE SELECTION}

An overview of the COSMOS program is given in Scoville et al. (2007a) and details of the Hubble Space Telescope (HST) observations are described in Scoville et al. (2007b). The COSMOS observations are undertaken in the F814W filter ("I band") and reach a depth of $I_{\mathrm{AB}}>27(10 \sigma)$. The photometric catalog and redshift measurements used in this paper are given in Mobasher et al. (2007) and Capak et al. (2007).

The most important and difficult step in studying the evolution of galactic structures is choosing comparable samples at different look-back times. For nearby galaxies, multiwaveband data with sufficient spatial resolution are available. Therefore galaxy properties (e.g., Hubble type, inclination, distance) are accurately known for nearby spirals. The underlying disk is also well imaged, and multiple techniques for identifying a bar may be employed. However, for high-redshift galaxies, the situation is more complicated. Here we summarize the steps we have taken to overcome these problems.

We choose all galaxies brighter than $L_{V}^{*}$ with an empirically determined luminosity evolution of 1 mag from Capak (2003) such that $M_{V}^{*}=-21.7$ at $z=0.9$ (Capak 2003). This criterion is specifically targeted for choosing galaxies from the same portion of the galaxy luminosity distribution at all redshifts. As we shall see later, a no-evolution luminosity model would have only steepened the overall decline in the bar fraction, further strengthening the results presented in this paper. For this luminosity criterion, at a redshift of $z=0.9$, the sample is complete for all galaxies with a half-light radius smaller than $10 \mathrm{kpc}$ (see Scoville et al. 2007b, Fig. 6). The number of galaxies with a half-light radius larger than $10 \mathrm{kpc}$ is extremely low (e.g., Fig. 10 in Sargent et al. 2007, or Fig. 10 in Barden et al. 2005) and thus our sample is essentially complete.

We eliminate all elliptical and lenticular galaxies based on the galaxy's spectral energy distribution (SED) type $T_{\text {phot }}$, choosing all galaxies with $T_{\text {phot }}>2 . T_{\text {phot }}$ is the best-fit spectral template ordered by the $4000 \AA$ A break strength (see Mobasher et al. 2007). The $T_{\text {phot }}$ sequence can be thought of as a photometric Hubble type going from the reddest early-type $\left(T_{\text {phot }}=1\right)$ galaxies to bluest late-type $\left(T_{\text {phot }}=6\right)$ galaxies. Types 1 through 4 are defined by the templates from Coleman et al. (1980) and correspond to elliptical, Sbc, Scd, and Irregular Hubble types, respectively. Types 5 and 6 correspond to Kinney et al. (1996) type SB3 and SB2, respectively, which are local starburst galaxies with little or no extinction. Typical uncertainties in $T_{\text {phot }}$ are \pm 0.2 .

The $T_{\text {phot }}$ values are robust descriptors of galaxies to $z \sim 1.2$ as confirmed by a comparison of the photometric and spectroscopic redshifts for over eight hundred galaxies (Mobasher et al. 2007). Comparison of galaxy types based on morphological parameters such as Gini and asymmetry and the $T_{\text {phot }}$ type shows that $T_{\text {phot }}>2$ selects all spirals to $z \sim 1.2$ (Capak et al. 2007; O. Ilbert et al., in preparation). With increasing redshift there is an increase in the population of blue ellipticals and bluebulge-dominated spirals; however, these galaxies are only significant at the faint end of the luminosity function (e.g., Ilbert et al. 2006; Capak et al. 2007; O. Ilbert et al., in preparation). For instance, at $M_{B}<-20$, the blue-bulge-dominated spiral population is less than $1 \%$ of the total disk population (Ilbert et al. 2006). At $z \sim 0.8$, among elliptical galaxies (identified by the Gini and asymmetry parameters), the contribution of blue ellipticals to the total volume density is significant at the very low mass end $\left(\sim 10^{9} M_{\odot}\right)$. For the high-luminosity (massive) galaxies, which are studied in this sample, the contribution from blue ellipticals is no more than a few percent. We do find a small fraction of compact systems with blue colors which we identify and reject from our analysis.

We impose a redshift cutoff of $z=0.835$ so that the F814W filter does not probe bluer than rest-frame $g$ band. The rationale for probing no bluer than rest-frame $g$-band is described in detail in Appendix A.1, but we also note here that at this redshift the angular diameter of a $0.05^{\prime \prime}$ ACS pixel subtends a physical scale of $0.4 \mathrm{kpc}$. At this resolution we expect to detect easily the entire population of bars in nearby spiral galaxies (see Fig. 3 of Sheth et al. 2003, or Fig. 7 of Menéndez-Delmestre et al. 2007). We note that all our galaxies subtend at least 10 pixels. The smallest Petrosian radius in our sample is 5.2 pixels.

Imposing these cuts reduces our sample to 3886 spirals. However, galactic structures such as bars are difficult to identify and quantify in edge-on galaxies. Therefore, we further eliminate all galaxies with inclinations $i>65^{\circ}$, the same limiting inclination used in studies of the bar fraction in nearby spirals (e.g., 
Menéndez-Delmestre et al. 2007). Inclination values for our sample are determined from the axial ratio of the galactic disk, which is identified using a two-dimensional decomposition with a bulge and exponential component for each galaxy using GALFIT (Peng et al. 2002). The inclination cut eliminates an additional 986 objects, leaving 2900 galaxies.

Finally, we discard all galaxies that were obviously merging, or too irregular or peculiar to be fit with ellipses. This eliminates 743 galaxies, leaving a final sample of 2157 spiral galaxies, which were then classified as barred or unbarred. The importance of eliminating peculiar objects from our analysis is investigated further in Appendix A.2, but we note here that including these objects would not have changed any of our conclusions.

All galaxies are detected in at least 13, sometimes all 16 available photometric bands. The galaxy luminosities, masses, and colors are measured from the photometric redshift code (Mobasher et al. 2007), which simultaneously solves for observed galaxy flux, redshift, galaxy type, and extinction intrinsic to the galaxy. The galaxy type combined with the flux-normalization yields rest-frame, extinction-corrected, luminosities and colors for each object. The rest-frame color and luminosity is then converted into stellar mass using the Bell et al. (2005) relation. Errors in the mass estimate are within a factor of 3 due to systematics. With our deep multiwavelength photometry these parameters are robust and nondegenerate at $z<1.2$. Over this redshift range we have at least two data points redward of the $4000 \AA$ break $\left(z^{+}\right.$and $K_{s}$ at $\left.z=1.2\right)$ and two points blueward $\left(u^{*}\right.$ and $B_{J}$ at $\left.z=0.3\right)$, which allows us to break the $4000 \AA$ break strength-extinction degeneracy. More detailed SED fits using spectroscopic redshifts yield similar results for the galaxy luminosity, mass, and color (Mobasher et al. 2007; O. Ilbert et al., in preparation).

\section{BAR CLASSIFICATION METHODOLOGY}

We identified bars using two methods and the results were cross-checked for consistency. We use the same methods for the local SDSS calibration sample (see Appendix A.1) and the COSMOS data to reduce biases that can be induced by the use of different classification methods.

Our first method was to use the ellipse-fitting technique that has been used widely by multiple studies of both nearby and high-redshift galaxies (e.g., Regan \& Elmegreen 1997; Sheth et al. 2000, 2002, 2003; Knapen et al. 2000; Laine et al. 2002; Jogee et al. 1999,2004; Barazza et al. 2008; Menéndez-Delmestre et al. 2007; Marinova \& Jogee 2007). For a detailed discussion of the ellipse-fitting method and the classification scheme we refer the reader to Menéndez-Delmestre et al. (2007). Briefly, bars are identified from a dual signature of the ellipticity and position angle profiles. We require that the ellipticity increases monotonically, exceeds 0.2 , and then drops by at least $\delta \epsilon>0.1$. The position angle profile should show a relatively constant position angle over the bar region and change by at least $10^{\circ}$ after the bar as the isophotes enter the disk. In general the fitting procedure is extremely robust and fits ellipses to the $1 \sigma$ noise level in the images - in the case of COSMOS this means we are able to fit galaxies to the outermost disk isophotes (at $\mu=24.5 \mathrm{mag} \mathrm{arcsec}^{-2}$; see Fig. 9) in the highest redshift bins. In a small number of cases, the ellipse-fitting method can miss an existing bar (see Fig. 3 in Menéndez-Delmestre et al. 2007). This usually happens when the position angle of the disk and bar are aligned or when the ellipticity drop is moderated by open spiral arms.

In addition to the ellipticity and position angle profiles, we classified the bars further into strong bars and intermediate bars by visually examining the isophote shapes. The strong bars are those with an ellipticity greater than 0.4 to be consistent with previous work in this field (e.g., Jogee et al. 2004). However, we note that the definition is arbitrary because there is a continuum of bar strengths (Block et al. 2002; Whyte et al. 2002; Buta et al. 2004, 2005; Menéndez-Delmestre et al. 2007). We refer to this first method as the "ElPa" classification method for the remainder of the paper.

Our second method for classifying galaxies was visual identification of galaxies into categories of SA (unbarred), SAB, and SB by one of the authors (D. M. E.). Not all galaxies were classified into these three neat categories. D. M. E. also classified galaxies as edge-on, clump-cluster galaxy, or compact (spheroidal) galaxy following her work on the Ultra Deep Field (Elmegreen \& Elmegreen 2005, 2006; Elmegreen et al. 2005a, 2005 b 2007). Galaxies that were visually classified as edge-on, clump-cluster, or compact are not included in the visual classification results. The total number of galaxies classified visually into barred and unbarred spirals is 1,705 .

The classification by D. M. E. was cross-checked for 500 galaxies by another author (K. S.), and also cross-checked against the ellipse-fitting method. The cross checks between the two methods finds agreement for $85 \%$ of the sample. In general we found that we classified more galaxies as barred by eye than using the ellipse-fitting profile. This is as expected because there are particular morphologies where the ellipticity and position angle signature can be masked by the relative orientation of the bar and disk, and the pitch angle of the spiral arms. As noted earlier, a detailed discussion of such cases can be found in Menéndez-Delmestre et al. (2007). Only in 5\% of the cases is there a gross mismatch where one method differed from the other by two classes, i.e., bar in one and spiral in the other. This generally occurred for very faint or small nuclei where the ellipse-fitting method has problems fitting the isophotes. In the remaining $10 \%$ of the cases, the methods agreed to within one class, i.e., intermediate bar in one, and a spiral or strong bar in the other. The cross checks were internally consistent at all redshifts.

\section{RESULTS}

\subsection{The Declining Bar Fraction}

Figure 1 shows the evolution of the bar fraction in COSMOS as a function of redshift in six equal redshift bins $(\delta z=0.117)$, starting at $z=0.14$ at a look-back time of $1.8 \mathrm{Gyr}$. The redshift bins correspond to look-back times of 1.8-3.0, 3.0-4.1, 4.1-5.0, 5.0-5.8, 5.8-6.5, and 6.5-7.1 Gyr, respectively. The two rows show the bar fractions measured from the two classification methods described above ( $(3)$. The left column shows the total bar fractions (strong bars + oval bars) and right column shows the strong-bar fraction (SB). For each data point we list the number of bars and the total number of galaxies classified in each bin. The error bars reflect the statistical uncertainty in the fraction and are calculated from the expression $\left[f(1-f)^{/ / N}\right]^{1 / 2}$ for fraction $f$ and number of galaxies $N$.

We find that the bar fraction (for all galaxy luminosities combined) has evolved dramatically over the last 7 Gyr. At $z=0.84$, the total bar fraction using the ElPa classification method is $f_{\text {bar }}=0.22 \pm 0.02(0.31 \pm 0.02$ for visual classification method $)$, one-third (one-half) its present-day value. We see the same trend when we consider only the strong bar fraction: $f_{\mathrm{SB}}$ evolves from $0.27 \pm 0.05(0.35 \pm 0.05)$ at $z=0.0$ to $0.09 \pm 0.01(0.17 \pm$ $0.02)$ at $z=0.835$.

When combined with the $z=0.0$ data point from our analysis of a local SDSS sample (Table 1, Fig. 6, and Appendix A.1), we also find that the evolutionary trend is weaker in the first three bins at $z<0.4$. Within the error bars, the data at these redshifts 


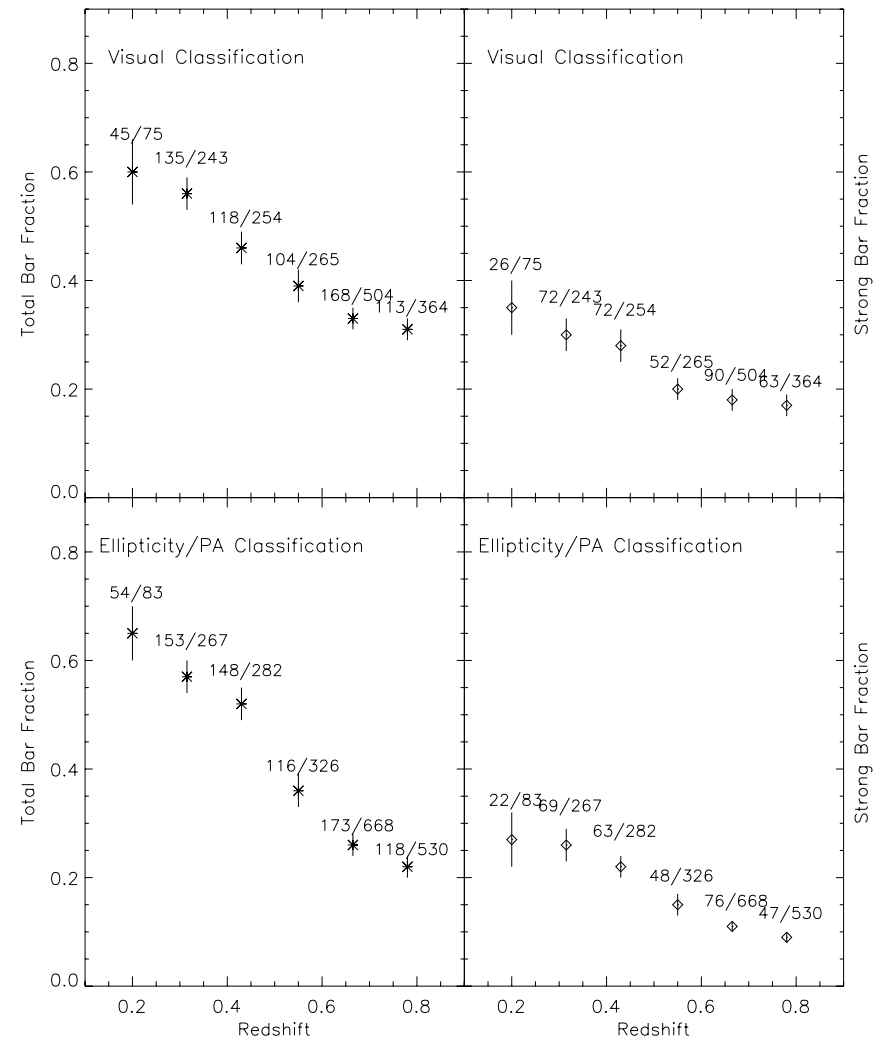

FIG. 1.-Evolution of the bar fraction as a function of redshift in equal bins from $z=0.0$ to $z=0.84$, out to a look-back time of $7 \mathrm{Gyr}$. The bar fraction drops from $65 \%$ in the local universe to about $20 \%$ at $z \sim 0.84$. The fraction of strong bars (SBs) drops from about 30\% to under $10 \%$. The top row shows the results from the visual classification and the bottom row shows the results based on classification using the ellipticity and position angle profiles. The left column shows the bar fraction for all galaxies classified as bars, whereas the right column shows the same only for the strong bars. The error bars are calculated as $[f(1-f) / N]^{1 / 2}$, where $f$ is the fraction of galaxies, and $N$ is the number of galaxies in a given category. The numbers above each data point show the total number of bars (or strong bars)/total number of galaxies in the bin.

are consistent with a roughly constant bar fraction $\left(f_{\mathrm{bar}}=0.6\right.$, $\left.f_{\mathrm{SB}}=0.3\right)$. These results are summarized in Table 1 .

\subsection{Bar Fraction as a Function of Galaxy Mass \& Luminosity}

Figures 2 and 3 show $f_{\text {bar }}$ versus the absolute luminosity and mass of the disk, respectively, in the three redshift bins from $z=0.14$ to $z=0.84$. We find that in the highest redshift bin, galaxies with masses $\log M\left(M_{\odot}\right)>10.9$ and luminosities $M_{V}<$ -23.5 have $f_{\text {bar }} \sim 0.5$, which is about the local value. In contrast, the low-mass $(\log M<10.5)$ and low-luminosity $\left(M_{V}>-22.5\right)$ galaxies have $f_{\mathrm{bar}}<0.2$ at high redshift. The same trend is seen for strong bars, $f_{\mathrm{SB}}$. At low redshifts, the bar fraction is roughly equal for all luminosities.

This trend is not due to incompleteness in the sample. We establish the completeness of our sample by measuring the mass limit based on our selection criteria. Since we choose galaxies based on a luminosity cutoff and galaxy colors, the mass completeness is most likely to be an issue for the reddest systems at the highest redshift. For our luminosity cutoff and $T_{\text {phot }}$ criteria, our sample is complete for galaxies with masses greater than $(3-4) \times 10^{10} M_{\odot}$ at $z=0.9$ for the reddest $\left(T_{\text {phot }}=2\right.$, rest-frame $\left.\Delta m_{g-r}>0.56\right)$ galaxies. Obviously, for the bluest systems (e.g., $\left.T_{\text {phot }}=6\right)$, our sample is complete to $(0.9-1) \times 10^{10} M_{\odot}$. These values are calculated from the Maraston (2005) and Bell et al. (2005) models, respectively. Note that at $z=0.6$, the mass limit for completeness in the sample is lowered by another $\sim 25 \%$.
Our lowest mass data point in the $0.6<z<0.84$ bin (the highest redshift bin) in Figure 3 is for galaxies with masses between $(3-4) \times 10^{10} M_{\odot}$. It is therefore free from the possible mass selection bias. The data points at lower masses and lower redshifts are also computed from a complete sample of masses for a given bin. Thus we conclude that the observed strong correlation between the bar fraction and mass in the highest redshift bin is a robust result.

The most important result in these figures is that in the highest redshift bins in this study, a majority of the most massive and luminous systems are barred. There is little evolution in the bar fraction with redshift in these systems. Since bars form in massive, dynamically cold and rotationally supported galaxies, the high bar fraction indicates that the most massive systems are already "mature" enough to host bars. This agrees with the analysis of the evolution of the size function of disk galaxies of several studies (Sargent et al. 2007; Ravindranath et al. 2004; Barden et al. 2005; Sheth et al. 2008), which find that large disks are already in place by $z=1$ and little or no evolution in disk sizes from $z \sim 1$ to the present epoch. Conversely the low bar fraction in the lower luminosity, lower mass systems indicates that these systems are either dynamically hot, not rotationally supported and/or have not accreted sufficient mass to host bars. Merging activity, which is more common at higher redshifts, is also likely to affect the less massive systems more severely and may be responsible for heating them up more than high-mass systems. Bar formation may be delayed in these hot disks if they are embedded in a massive dark matter halo. Although the exact nature of these disks is not yet well known, there is an indication that later type systems may be dynamically hotter (Kassin et al. 2007). We consider these points further in following sections.

\subsection{Bar Fraction as a Function of Galaxy Color and Bulge Luminosity}

Figure 4 shows how $f_{\text {bar }}$ varies with galaxy SED type $\left(T_{\text {phot }}\right)$ and redshift. At low redshift, $f_{\text {bar }}$ is independent of $T_{\text {phot }}$, and at high redshift, $f_{\text {bar }}$ decreases from early $\left(T_{\text {phot }}<3\right)$ to late types $\left(T_{\text {phot }}>3\right)$. Similarly, $f_{\text {bar }}$ decreases with redshift more strongly for the late types than the early types. This latter trend is consistent with the previous result that the bar fraction changes with redshift primarily for the low-mass galaxies, which tend to have late SED types.

Finally we consider how the bar fraction varies as a function of the bulge light in galaxies. Figure 5 shows $f_{\text {bar }}$ versus the fraction of bulge luminosity in a galaxy for different redshift bins. The bulge magnitude is calculated from fitting each galaxy with a Sérsic+exponential profile using GALFIT (Peng et al. 2002). The $x$-axis in this figure is the difference between the bulge magnitude measured from the GALFIT fitting and the total (disk + bulge) apparent magnitude. We note that the relative calibration across redshift bins should be treated with caution because we are not correcting for $k$-correction effects that are known to affect two-dimensional decomposition of galaxies. Within a given redshift bin, however, the bulge contribution measurement should be robust except for one important caveat. The fitting algorithm is not designed to decompose a bar separately. As a result the bar light is likely to be split between the exponential and Sérsic components. If the light profile of a bar is exponential, as it is in later Hubble-type galaxies locally, the majority of that light is likely to be part of the exponential component. On the other hand, if the bar is relatively short and not highly elliptical, its light is likely to be added to the Sérsic component. The detailed decomposition of the bulge+bar+disk will require a more sophisticated approach, which is beyond the scope of this paper. 
TABLE 1

Galaxy Classification and Bar Fraction with Redshift

\begin{tabular}{|c|c|c|c|c|c|c|c|c|c|}
\hline$z_{L}$ & $z_{U}^{\mathrm{a}}$ & $N_{\text {bin }}^{\mathrm{b}}$ & $\mathrm{SP}_{\text {bin }}{ }^{\mathrm{c}}$ & $\mathrm{WB}_{\text {bin }}{ }^{\mathrm{d}}$ & $\mathrm{SB}_{\text {bin }}{ }^{\mathrm{e}}$ & $f_{\text {bar }}^{\mathrm{f}}$ & $1 \sigma^{\mathrm{g}}$ & $f_{\mathrm{SB}}{ }^{\mathrm{h}}$ & $1 \sigma^{\mathrm{g}}$ \\
\hline \multicolumn{10}{|c|}{ Visual Classification } \\
\hline 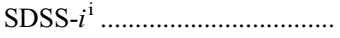 & 0.00 & 139 & 57 & 23 & 59 & 0.59 & 0.04 & 0.42 & 0.04 \\
\hline 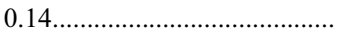 & 0.26 & 75 & 30 & 19 & 26 & 0.60 & 0.06 & 0.35 & 0.05 \\
\hline 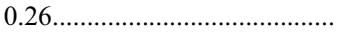 & 0.37 & 243 & 108 & 63 & 72 & 0.56 & 0.03 & 0.30 & 0.03 \\
\hline $0.37 \ldots \ldots \ldots$ & 0.49 & 254 & 136 & 46 & 72 & 0.46 & 0.03 & 0.28 & 0.03 \\
\hline 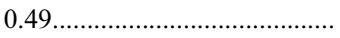 & 0.61 & 265 & 161 & 52 & 52 & 0.39 & 0.03 & 0.20 & 0.02 \\
\hline 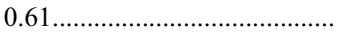 & 0.72 & 504 & 336 & 78 & 90 & 0.33 & 0.02 & 0.18 & 0.02 \\
\hline 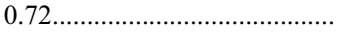 & 0.84 & 364 & 251 & 50 & 63 & 0.31 & 0.02 & 0.17 & 0.02 \\
\hline \multicolumn{10}{|c|}{ Ellipse/P.A. Classification } \\
\hline SDDS- $i^{\mathrm{i}} \ldots$ & 0.00 & 139 & 57 & 26 & 56 & 0.59 & 0.04 & 0.41 & 0.04 \\
\hline 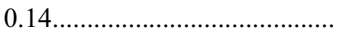 & 0.26 & 83 & 29 & 32 & 22 & 0.65 & 0.05 & 0.27 & 0.05 \\
\hline 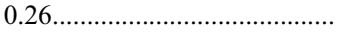 & 0.37 & 267 & 114 & 84 & 69 & 0.57 & 0.03 & 0.26 & 0.03 \\
\hline 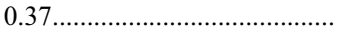 & 0.49 & 282 & 134 & 85 & 63 & 0.52 & 0.03 & 0.22 & 0.02 \\
\hline 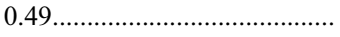 & 0.61 & 326 & 210 & 68 & 48 & 0.36 & 0.03 & 0.15 & 0.02 \\
\hline 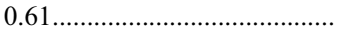 & 0.72 & 668 & 495 & 97 & 76 & 0.26 & 0.02 & 0.11 & 0.01 \\
\hline 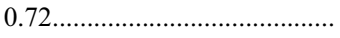 & 0.84 & 530 & 412 & 71 & 47 & 0.22 & 0.02 & 0.09 & 0.01 \\
\hline
\end{tabular}

${ }^{a}$ Upper limit of redshift bin.

b Total number of spiral galaxies in bin.

c Number of galaxies classified as unbarred in bin.

d Number of galaxies classified as weak bars (SAB) in bin.

e Number of galaxies classified as strong bars (SB) in bin.

${ }^{f}$ Equals $\mathrm{WB}_{\text {bin }}+\mathrm{SB}_{\text {bin }} / N_{\text {bin }}$.

Equals $\mathrm{SB}_{\text {bin }} / N_{\text {bin. }}$.

h Error assuming binomial statistics, $1 \sigma=f(1-f) / N_{\text {bin }}$, where $f$ is $f_{\text {bar }}$ or $f_{\mathrm{SB}}$.

${ }^{\mathrm{i}}$ Lower limit of redshift bin is denoted by $i$.

Keeping the above caveat in mind, we find that $f_{\text {bar }}$ is slightly higher for galaxies that are "bulge dominated" compared to galaxies that are not bulge dominated in the highest redshift bin. In the lowest redshift bin the slight trend disappears and the bar fraction is roughly constant for all types of bulges, although there are only a few galaxies that are not bulge dominated. This correlation together with the previous correlations (Figs. 2, 3, and 4) suggests that the galaxies that are red, luminous, and massive are also bulge dominated, and in these galaxies the bar fraction does not vary strongly with redshift. We discuss the implications of the bar-bulge correlation in $\S 5.4$.

\section{DISCUSSION}

\subsection{Comparison with Previous Studies}

In Figure 6 we plot all of the data from previous studies of the evolution of the bar fraction for comparison to the COSMOS results. In all cases there is a general decline in the bar fraction. However, the interpretation of the data has been very different among these studies as we discuss below.

Our basic result of a decline in the bar fraction is consistent with the earliest HDF studies (Abraham et al. 1996, 1999; van den Bergh et al. 1996) shown with the red data points in Figure 6. These studies reported a striking decline in the fraction of bars at $z>0.5$. But reanalysis of their Figure 4 shows that, in fact, at $z>0.5$, there are 10 barred spirals out of 29 galaxies, and at $z>0.8$, there are three barred spirals out of 11 galaxies, consistent with the COSMOS results presented here. Similarly our result is consistent with the very low bar fraction $(5 \%-10 \%)$ measured from the NICMOS HDF (Sheth et al. 2003) at $z>0.7$. But it is difficult to compare this bar fraction to the ours because the NICMOS data can only probe the largest bars. Of course the volume probed by the HDF studies was too small to allow evolution in $f_{\text {bar }}$ to be probed with much confidence.
On the other hand, our central result is fundamentally different from that reported by Jogee et al. (2004) and Elmegreen et al. (2004); these studies have reported a constant bar fraction with redshift to $z \sim 1$. In the Jogee et al. (2004) study, the authors classified 258 galaxies as either strongly barred or unbarred. Their modest sample size, however, prevented them from studying the $f_{\text {bar }}$ evolution in detail. Their first bin, for example, encompasses our central four redshift bins. Nevertheless we can compare their measurement of $f_{\mathrm{SB}}$ to ours. Over the same redshift range in the COSMOS data, we measure a strong bar fraction, $f_{\mathrm{SB}}=0.23 \pm 0.01$ for the visual classification, and $0.17 \pm$ 0.01 for the ElPa classification methods. In comparison, Jogee et al. (2004) reports a $f_{\mathrm{SB}} \sim 0.3 \pm 0.03^{13}$. Even though the Jogee et al. (2004) study probes fainter galaxies $\left(M_{V}<-19.3\right)$, which should have resulted in a lower bar fraction, their $f_{\mathrm{SB}}$ is $50 \%$ higher than the COSMOS results. Some possible reasons for the discrepancy in the value of $f_{\mathrm{SB}}$ may be the different use of inclination cuts (they used $i>60^{\circ}$ compared to our cut at $i>60^{\circ}$ ), an effectively different bar strength threshold, and different galaxy selection criteria. We chose our sample based on a galaxy luminosity with an evolving luminosity function, spectral type/ color and visual classification, whereas Jogee et al. (2004) chose their sample based on a fixed (lower) luminosity, range of $U-V$ colors (which is similar to our cut in $T_{\text {phot }}$ ), and a Sérsic parameter from a single fit to the galaxies or a concentration index. Given the various uncertainties at hand, we conclude that the data presented by Jogee et al. (2004) can be interpreted as being consistent with the more significant decline seen in our sample.

\footnotetext{
${ }^{13}$ Errors are not reported in the Jogee et al. (2004) study. We measure an error for their data using the bar fraction and total number of galaxies reported, in the same way as we measured for the COSMOS data.
} 


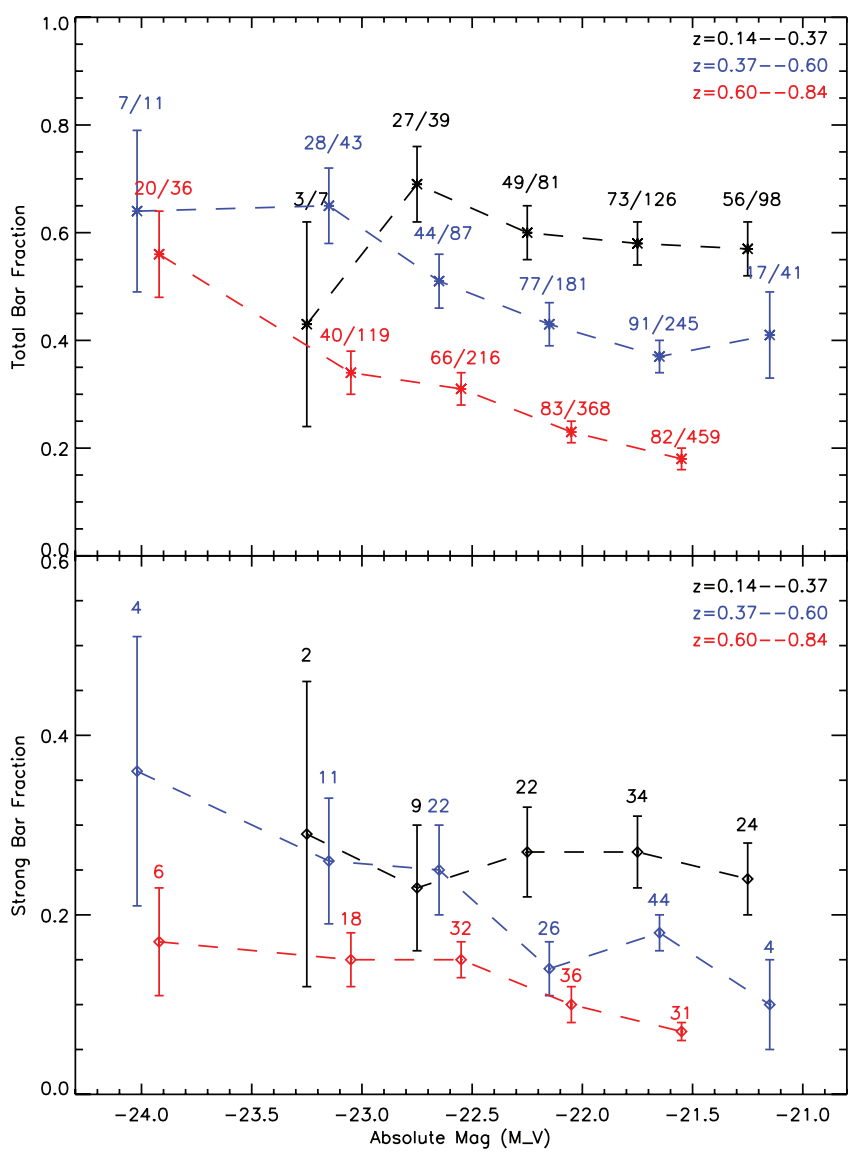

FIG. 2.- Total bar fraction (top panel) and strong bar fraction (bottom panel) vs. the absolute magnitude $\left(M_{V}\right)$ in three redshift bins. There is a strong correlation between the bar fraction and the galaxy luminosity in the highest redshift bin. In that bin, the most luminous galaxies $\left(M_{V}<-23.5\right)$ have $f_{\text {bar }} \sim 0.5$, whereas the lowest luminosity $\left(M_{V}>-22.5\right)$ galaxies have a $f_{\text {bar }} \sim 0.2$. With redshift we see a strong evolution in the low-luminosity sample as they evolve to $f_{\text {bar }} \sim$ 0.6 in the lowest redshift bin. A similar trend is seen in the bottom panels for the strong bar fraction. The data points are at the midpoints of bins of $\delta M_{V}=0.5$, from -21.0 to -23.5 (a data point is skipped if no galaxies are found in a bin) and the errors bars are calculated as before. The first bin is from $M_{V}=-23.5$ to -24.75 . The data points are slightly offset along the $x$-axis for each redshift bin and only the number of strong bars is labeled on the bottom panel for clarity. This figure should be viewed together with Fig. 3 .

Elmegreen et al. (2004) also reported a constant bar fraction to $z \sim 1.1$ based on an analysis of 186 background galaxies larger than 10 pixels in diameter in the multicolor ACS image of the Tadpole galaxy. These data points are shown with the purple triangles in Figure 6 . The data show a declining bar fraction from $\sim 30 \%$ to $\sim 15 \%$ out to $z=0.8$ with $3 \sigma$ uncertainty, and a rise in the bar fraction from $z=0.8-1.1$, which is beyond the redshift investigated here. ${ }^{14}$ Their conclusion that the bar fraction is flat on average followed primarily from the second rise at $z \sim 1$; otherwise their fractions agree with ours to within statistical uncertainties. Our results are also in line with a recent analysis of the Hubble Ultra Deep Field where the bar fraction, shown with the blue triangle, is $\sim 10 \%$ (Elmegreen et al. 2005a) at $z \sim 1$, consistent with the previous HDF studies and the values obtained in this paper.

Although we have attempted to put all the data from various studies into context, we emphasize that it is not straightforward to make direct comparisons because of different selection criteria

14 Four galaxies in their Fig. 10 at $z \sim 1.8$ are incorrect because of a photometric redshift error-the corrected data point is shown here.

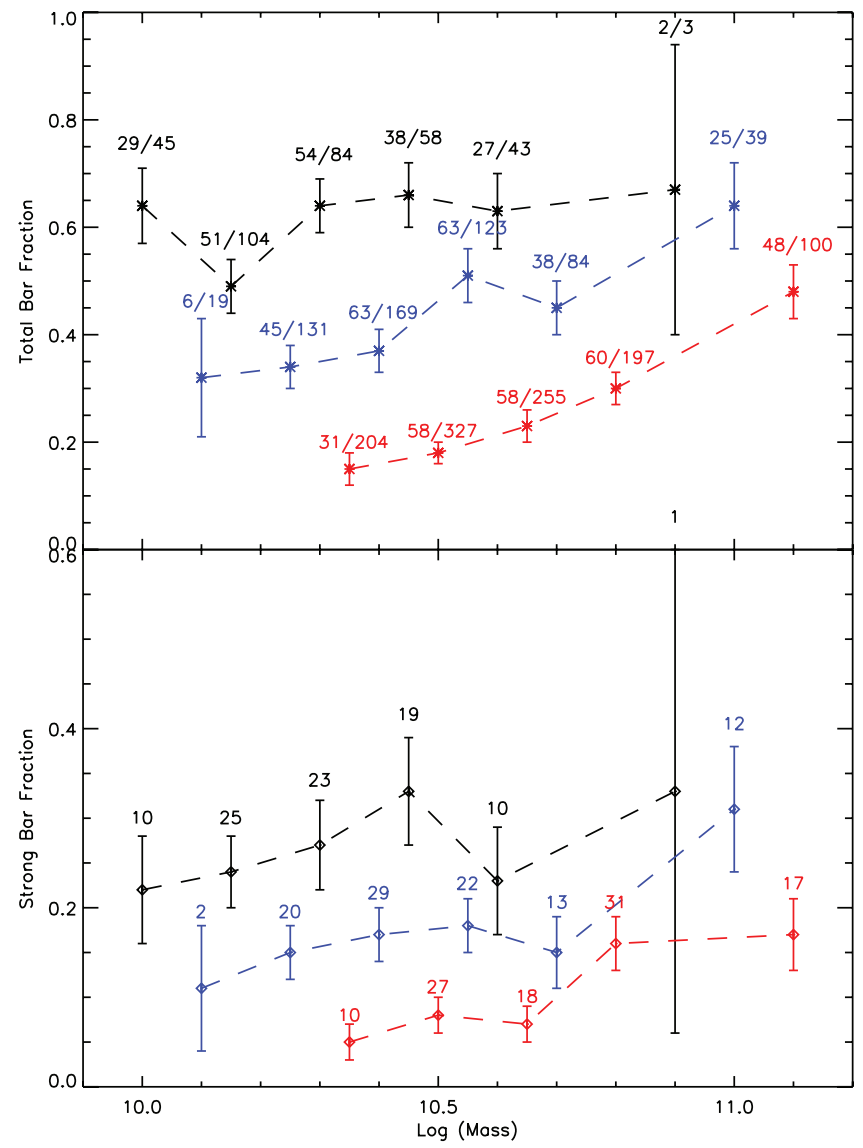

FIG. 3.- Total bar fraction (top panel) and strong bar fraction (bottom panel) vs. total galaxy stellar mass in three redshift bins. As expected from Fig. 2, there is a strong correlation between the bar fraction and the mass for the highest redshift bin. In that bin, galaxies with $\log M>10.9$ already have $f_{\text {bar }} \sim 0.5$, whereas galaxies with $\log M<10.5$ have $f_{\text {bar }}<0.2$. The bar fraction for the entire population evolves with time with the largest change in the lowest mass bin. The same trend is seen in the bottom panel. The lack of high-mass galaxies in the lower redshift bins is because, even with $2 \mathrm{deg}^{2}$, the volume of space observed by COSMOS is small. Luminosity and color selection criteria put a limit on the minimum detectable mass - our sample is complete for the points shown here. Each point is at the left edge of bins of $\delta \log M=0.15$, starting from 10.0 (point skipped if no data is found in a bin). Errors bars are calculated as before and last bin is from $\log M=$ 10.9-11.5. The data points are slightly offset along the $x$-axis for each redshift bin and only the number of strong bars is labeled on the bottom panel for clarity. The uncertainty in the mass measurement is a factor of three. This figure should be viewed together with Fig. 2.

and bar identification methods between these studies. These may be responsible for some of the observed differences. The main point to note is that in nearly every study, the data have shown a decline in the bar fraction, although the interpretations of the data have ranged from a constant bar fraction to a dramatic paucity of bars at $z \sim 1$. It is only with the COSMOS data set that we are able to robustly quantify the decline in the bar fraction and show that the evolution is a strong function of the galaxy luminosity, mass, color, and bulge dominance.

\subsection{Formation of the Hubble Sequence: Assembling the Spiral Galaxies}

The declining bar fraction reported in this paper shows that at a look-back time of $7 \operatorname{Gyr}(z=0.835)$ only about one-fifth of $L^{*}$ spiral galaxies were barred, which is about one-third the present day value. During the following $3 \mathrm{Gyr}$ (from $z \sim 0.8$ to $z \sim 0.3$ ) the bar fraction increased to roughly its present value. Only small changes occurred in the last 4 Gyr $(z<0.3)$. 


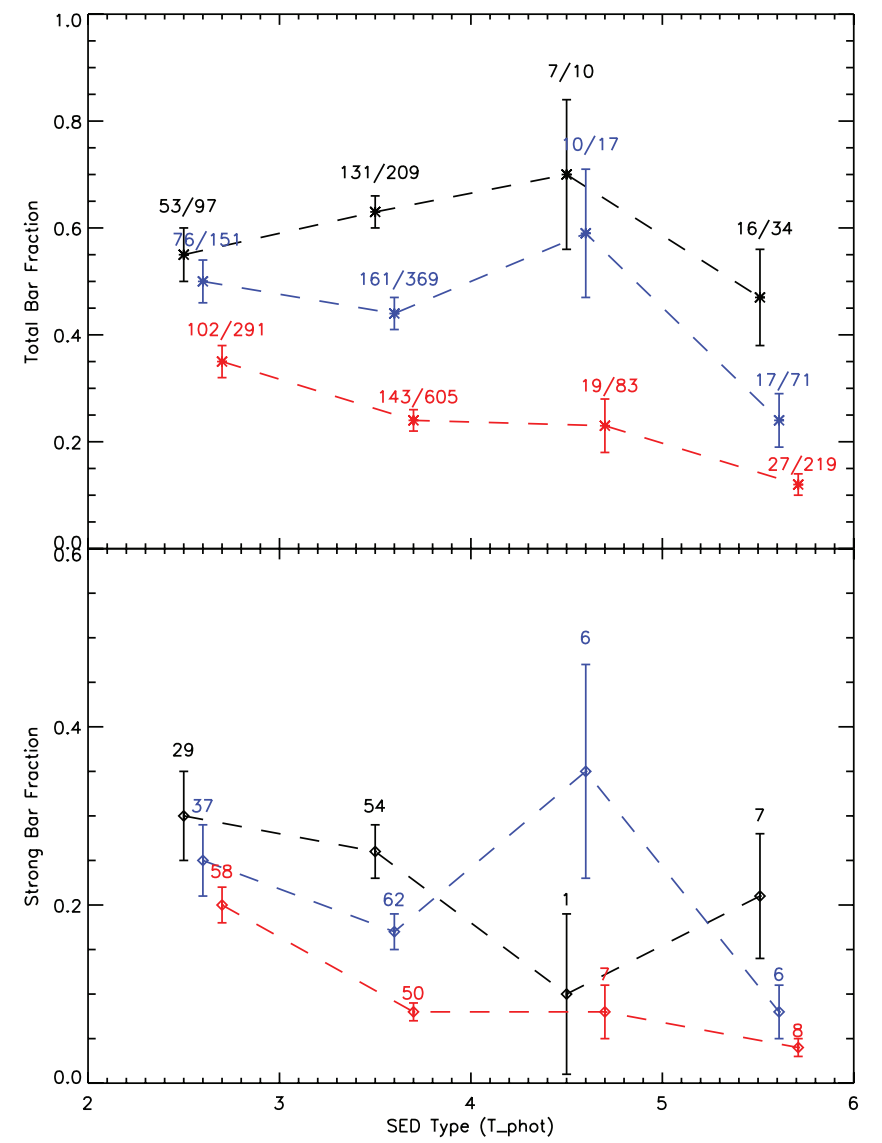

FIG. 4.- Total bar fraction (top panel) and strong bar fraction (bottom panel) vs. spectral type of a galaxy. In the highest redshift bin, the bar fraction decreases monotonically toward later spectral types. In contrast, in the lowest redshift bin the bar fraction is constant within the error bars across the $T_{\text {phot }}$ sequence. The data thus show that the majority of the evolution in $f_{\text {bar }}$ is in the bluer, later SED type systems. This is consistent with the results shown in previous figures for high- and low-mass galaxies, considering the usual correlation between mass and $T_{\text {phot. }}$ As before, the data points are slightly offset along the $x$-axis for each redshift bin and only the number of strong bars is labeled on the bottom panel for clarity.

This evolution can be understood within the framework of classical bar formation theory. $N$-body simulations have long suggested that bars form spontaneously in galactic disks, usually on relatively fast dynamical timescales. There are, however, two ways of slowing this down. One is to increase the halo mass fraction within the disk radius, and the other is to heat up the disk Athanassoula \& Sellwood (1986). Self-consistent threedimensional (3D) simulations essentially agree with this, although the role of the halo is now understood to be more complex, so that the final bar can be considerably stronger in cases where it grows slower Athanassoula $(2002,2003 a)$. Thus, the time it takes for an unbarred disk galaxy to become barred can vary widely. In cold, disk-dominated cases, the bar forms within a Gyr or less, but sufficiently hot disks embedded in very massive halos can stay unbarred several Gyrs. Such a delay might well explain the time evolution of the barred galaxy fraction shown in Figure 1. Furthermore, observations show that the halo-to-disk mass ratio is higher in low-mass, low-luminosity galaxies than in bright, massive galaxies (Bosma 2004; Kranz et al. 2003) so that bars are expected to grow later in the former, as we indeed find here. Hence if galactic disks are formed with a variety of velocity dispersions and a variety of halo-to-disk mass ratios, there should be a continuous increase with time in the barred fraction, as observed here. The slope of this evolution will depend on the dis-

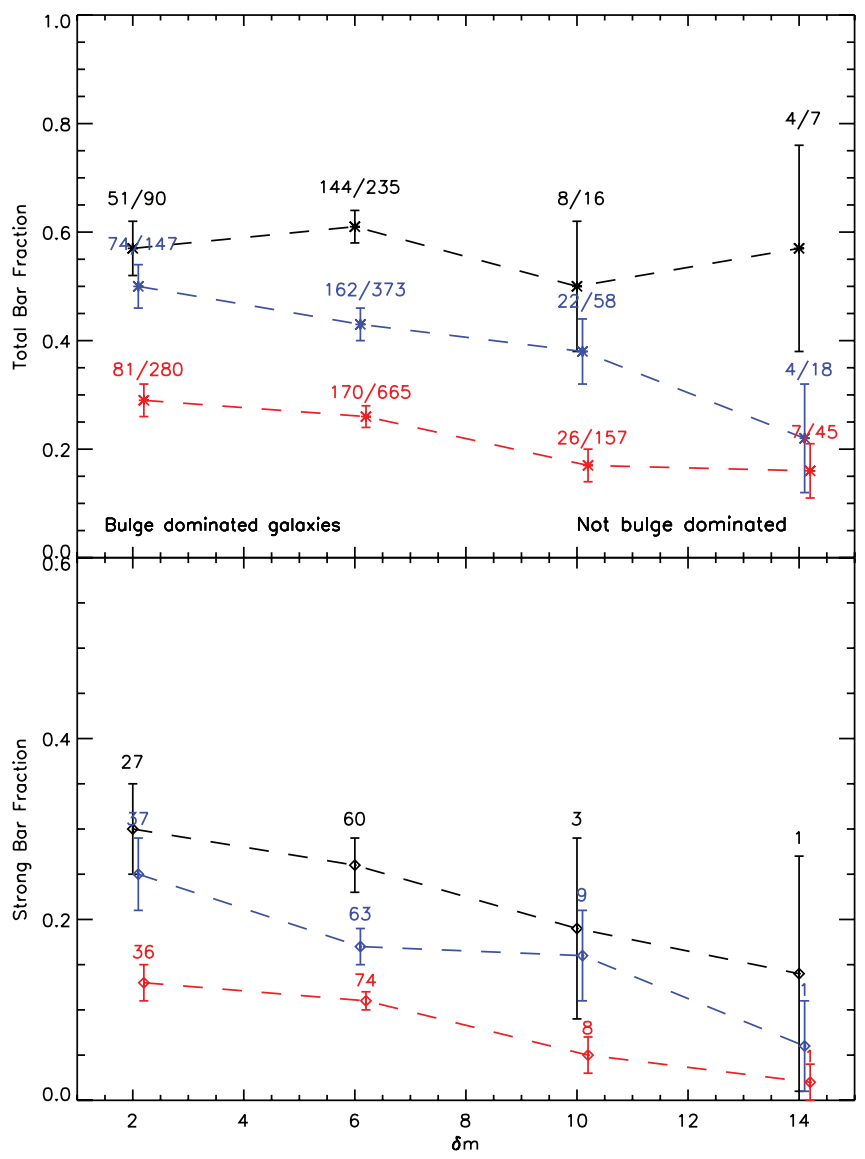

FIG. 5.- Bar fraction as a function of the bulge contribution in galaxies in three redshift bins, as before. We find that in the two high-redshift bins there is a slight preference for bars to be in bulge-dominated systems. The difference is less pronounced in the lowest redshift bin. The $x$-axis is calculated by subtracting the apparent bulge magnitude from the total galaxy magnitude. The bulge magnitude is calculated by fitting each galaxy with an exponential and bulge profile using GALFIT as discussed in the text. The $x$-axis thus indicates the fractional contribution of the bulge to the total luminosity of the galaxy with bulge-dominated systems to the left on the $x$-axis. Since $k$-corrections are important in two-dimensional decomposition of galaxies, the results across redshift bins are not as robust as those within a given redshift bin. The data points are at the midpoints of each $T_{\text {phot }}$ type bin, and as before, only the number of strong bars is listed in the bottom panel.

tribution of the initial disk and halo parameters. On the contrary, if all galaxy disks were, in the relevant time-period, similar, then the fraction of disk galaxies that are barred would be more or less constant with time, or show only a very small increase.

The preceding paragraphs assumed the existence of the appropriate set of physical conditions needed to form bars from the classical disk instability. Alternatively, bar formation may coincide with inner disk growth. This, however, would imply considerable growth of the inner disk even for $z \sim 0.84$, which seems inconsistent with recent results that show no evolution in the disk scale-lengths at $z<1$ (Ravindranath et al. 2004; Barden et al. 2005; Sargent et al. 2007; Sheth et al. 2008). A further point to take into consideration is that bars may dissolve when a gaseous component is included in the angular momentum exchange cycle, and/or in the presence of a sufficiently massive, centrally concentrated object, as e.g., a black hole (Friedli \& Benz 1993; Berentzen et al. 1998; Fukuda et al. 2000; Bournaud \& Combes 2002; Shen \& Sellwood 2004; Athanassoula et al. 2005; Bournaud et al. 2005). Observational evidence for bar dissolution, however, is at present rather sparse (Das et al. 2003; Block et al. 2002), while the amount of mass necessary seems to be larger than what is currently observed for central mass concentrations 


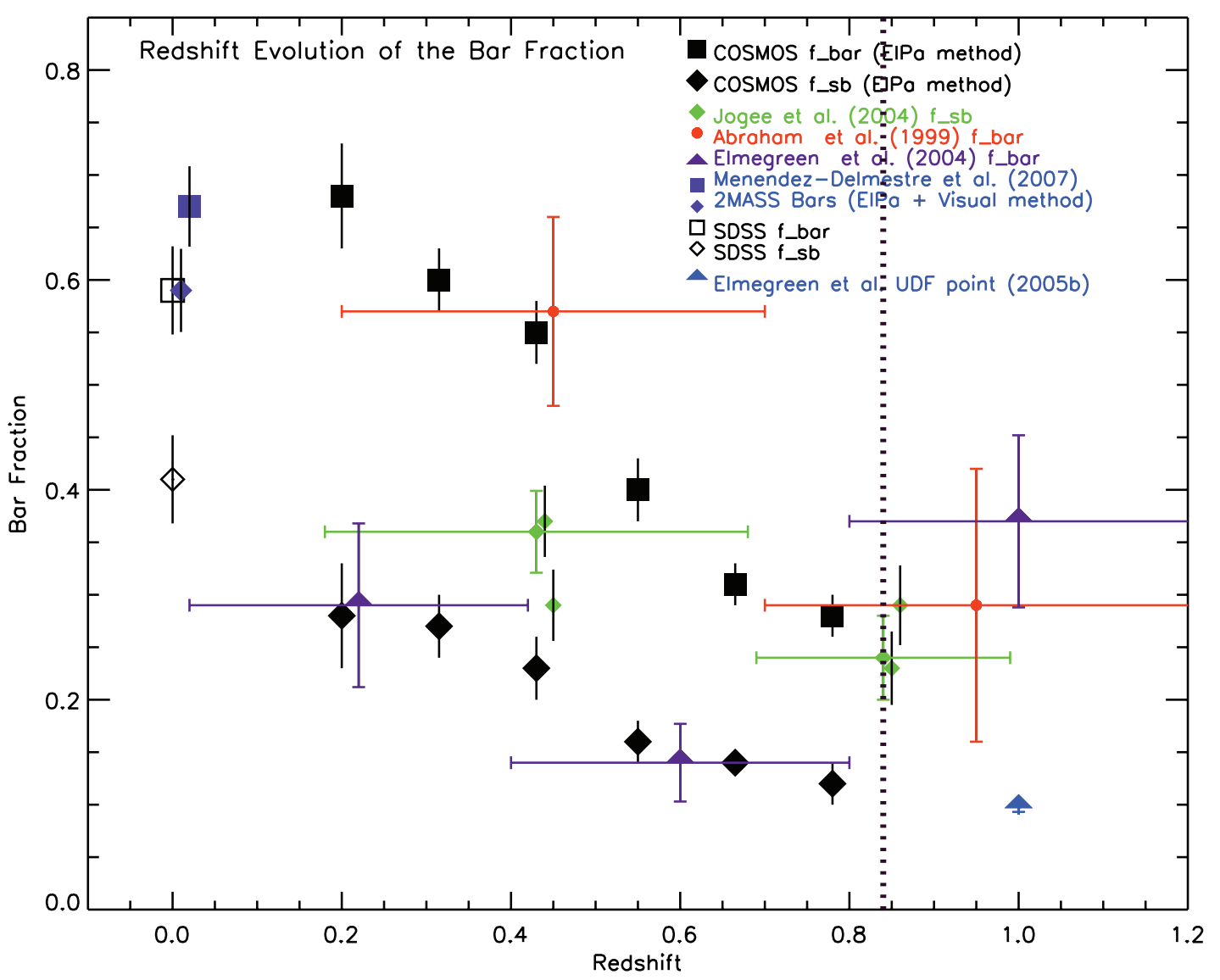

FIG. 6. - Comparison of measurements of our bar fraction with previous studies. $f_{\mathrm{bar}}$ and $f_{\mathrm{SB}}$ measured using the EIPa method for the COSMOS data are shown with the black filled squares and diamonds respectively. The red data circles (13/30 bars in low z bin, 4/14 in the high-z bin) are from Abraham et al. (1999); these do not distinguish between weak and strong bars. Purple triangles are bars and twists from Elmegreen et al. (2004) (10/34, 13/90, 13/35 bars, respectively); we summed adjacent bins from their data; they suggested inclination and resolution effects should increase the fractions by about a factor of 2. The green points from Jogee et al. (2004) are the strong bar fractions from GEMS; the three points in the two redshift bins are not independent of each other - they are measured for $\sim 110-175$ galaxies, chosen in different ways from the same sample. The horizontal bars show the redshift range over which these data are averaged. Also shown are data from our analysis of a SDSS control sample (square - $f_{\text {bar }}$, diamond $-f_{\mathrm{SB}}$ ), and from the 2MASS survey by Menéndez-Delmestre et al. (2007) (blue diamond is when both ellipticity and position angle signatures are present, whereas the square also includes candidate bars. The vertical dotted line is the limiting redshift for our survey. Within the error bars all the data seem to be in agreement. Contrary to earlier interpretations, it seems that all studies show a general decline in the bar fraction with redshift. It is only with the COSMOS data that we are able to analyze this decline in detail.

(Shen \& Sellwood 2004; Athanassoula et al. 2005). Nevertheless, it is by no means clear that this mechanism is unimportant.

We have so far discussed only isolated galaxies. Let us now turn to the effect of interactions and mergings. The number of interactions are known to increase dramatically with redshift (e.g., Kartaltepe et al. 2007 and references therein). Interactions and merging activity are most likely to influence (heat up) the less massive galaxies. It is precisely in such galaxies that we see significantly lower bar fractions compared to the high-mass galaxies at the highest redshifts (Figs. 3 and 4). Although indirect, there is observational evidence that later type and less massive systems are dynamically hotter. The top row of panels in Figure 1 of Kassin et al. (2007) clearly shows that late-type spirals and irregulars have larger disordered motions compared to early-type spirals particularly at high redshifts. These are precisely the type of systems within which we find fewer bars. Moreover, in the same figure, the higher mass galaxies also have a higher fraction of ordered motions than disordered motion, although the trend is hard to see in the relatively modest sample size in the highredshift bins. These data suggest that the lack of bars may therefore be related to the dynamic hotness and the mass surface density of these disks. We are currently identifying bars and measuring the bar fraction in this sample of galaxies and should be able to provide a direct answer for the said hypothesis (Sheth et al. 2008).
Simulations show that interactions speed up bar formation in direct encounters, but have little effect in retrograde ones (Toomre \& Toomre 1972; Noguchi 1987; Gerin et al. 1990; Steinmetz \& Navarro 2002), in good agreement with observations (Kormendy \& Norman 1979; Elmegreen \& Elmegreen 1982). Thus one might have expected higher rates of bar formation at high $z$, where interactions are common. On the contrary, it is possible for mergings to destroy or severely weaken the bar, without destroying the disk (e.g., Berentzen et al. 2004 and references therein). More modeling needs to be done before we can say with any certainty what the combined effect of interactions and mergings is. Note that we discarded from our statistics obviously interacting systems based on tidal features or obvious distortions. However, if a galaxy is weakly interacting, it would be difficult to distinguish it from a noninteracting system; this is already the case even in the local universe. So our sample of galaxies is most likely probing quiescent, postmerger or weakly interacting disks.

\subsection{The Downsizing Signature in Formation of Galactic Structure}

Galaxy "downsizing" was coined by Cowie et al. (1996) to refer to an evolutionary history in which the most massive galaxies formed first. There is strong observational evidence for the 
downsizing phenomena: the presence of massive systems at high redshifts (e.g., Daddi et al. 2004, 2005; Scarlata et al. 2007a, $2007 \mathrm{~b}$ ), an order of magnitude decline in the typical star formation rate (Arnouts et al. 2005), a change in the star formation activity to lower mass systems with decreasing redshifts (Treu et al. 2005; Glazebrook et al. 2004; Fontana et al. 2004; Maier et al. 2005, 2006; Bundy et al. 2006), and the decrease in characteristic luminosity of active galactic nuclei at low redshifts (Pei 1995; Ueda et al. 2003).

The results presented here show a downsizing signature in the formation of bars. The most massive, luminous and red galaxies have a higher bar fraction in the highest redshift bin with the most massive and luminous systems having a bar fraction close to the present-day value. The early presence of bars in these galaxies in the context of bar formation ( $\$ 5.2$ ) suggests that these systems "matured" early, i.e., they became dynamically cool and sufficiently massive to host bars at $z>0$.8. In contrast, the lower mass systems which are also bluer, acquired a majority of their bars at $z \sim 0.8$. Thus the downsizing phenomenon must be considered to be an effect more fundamental than one concerned solely with the regulation of ongoing star formation; it seems to be intimately connected with the dynamical maturity of the stellar disk.

\subsection{The Coevolution of Bulges and Bars}

Figure 5 shows that in our highest redshift bin there is a somewhat higher fraction of bars in galaxies with more massive bulges. This is consistent with the structural downsizing discussed in the previous section because galaxies with bulges are denser and more evolved in the center than galaxies without bulges. Bars and bulges apparently form at about the same time, with later times for lower mass galaxies. Some care is necessary in the interpretation of this result, however, since bulges are an inhomogeneous class of objects. In this paper, we defined the bulge as the component in the central part that contributes extra light above an extrapolated exponential fit to the outer part. This definition includes both classical (3D) bulges and disklike pseudobulges (Kormendy \& Kennicutt 2004; Athanassoula 2005). Pseudobulges form by gas inflow and star formation. Because bars drive inflow (Sheth et al. 2003; Sakamoto et al. 1999), there should be a correlation between disky bulges and bars, independent of dynamical downsizing. Bars may also contribute to the growth of nuclear black holes if they correlate with bulges, because there is a tight correlation between black hole mass, stellar velocity dispersion, and luminosity of bulges (Kormendy \& Richstone 1995; Magorrian et al. 1998; Ferrarese \& Merritt 2000). It will be interesting to test such differences with subsequent analysis of the bulges and bars in the COSMOS data.

\section{CONCLUSIONS}

Bars are an important signpost of galaxy evolution because once a galaxy disk is sufficiently massive, dynamically cold and rotationally supported it forms a bar. Therefore the evolution of the bar fraction over time is an important indicator of the evolutionary history of disk galaxies and the assembly of the Hubble sequence. Using a detailed analysis of $2157 \mathrm{~L}^{*}$ face-on, spiral galaxies from $0.0<z<0.84$ in the COSMOS $2 \mathrm{deg}^{2}$ survey we have investigated the evolution of the bar fraction over the last 7 Gyr. We have undertaken an extensive and careful analysis of selection effects ( $k$-correction, surface brightness dimming, inclination, spatial resolution, etc.), which is detailed in Appendix A. Our main results are as follows:

1. The bar fraction for $L^{*}$ galaxies drops from about $65 \%$ in the local universe to about $20 \%$ at $z=0.84$. Over this redshift range the fraction of strong bars (SBs) drops from about $30 \%$ to under $10 \%$. Thus at a look-back time of $7 \mathrm{Gyr}$, when the universe was half its present age, fundamental aspects of Hubble's "tuning fork" classification sequence had not yet fallen into place. Only about one-fifth of all spiral galaxies were "mature" enough (dynamically cold, massive and rotationally supported) to host galactic structures of the type we see today.

2. For the total $f_{\text {bar }}(\mathrm{SB}+\mathrm{SAB})$, the change is far less dramatic between $z=0.3$ and $z=0.0$ indicating slow evolution in galactic structures in $L^{*}$ galaxies over the last $4 \mathrm{Gyr}$. It is likely that there is significant evolution in the formation of bars in the sub- $L^{*}$ galaxies over this period.

3. One of the most significant findings in this study is the correlation between $f_{\text {bar }}$ and the galaxy mass, luminosity and color. We find that in the highest redshift bins $f_{\text {bar }}$ is higher in the more massive, luminous and redder systems. In fact, in the most massive systems, $f_{\text {bar }}$ is already as high at $z=0.8$ as the local value. These systems thus had already arrived with their present Hubble types at a look-back time of 7 Gyr. In the subsequent $3 \mathrm{Gyr}$, from $z=0.84$ to $z=0.3$, the lower mass, bluer systems evolved more slowly toward their present Hubble types. Thus the signature of downsizing is intimately connected with dynamical maturity of disks and is present in the formation of galactic structure.

4. Finally, we find a slight preference for barred galaxies to be more bulge-dominated in the high-redshift bin. This correlation is consistent with the dynamical downsizing found for bars in general if bars and bulges both form earlier and more prominently in the most massive galaxies. The lack of a stronger correlation may be related to the variety of bulges: bars are also likely to be involved with the inflow that builds pseudobulges. Given the strong correlation between bulge properties and black hole mass seen today, there may be a coevolution of bars, bulges, and black holes in some galaxies. The exact details of these processes remain to be investigated.

We are indebted to the anonymous referee for many helpful comments and suggestions that have greatly improved this paper. We are also thankful for the insightful and helpful discussions we have had with Karin Menendez-Delmestre, Neal Evans, Lee Armus, Isaac Shlosman, Wyn Evans, Donald Lynden-Bell, Mark Dickinson, David Elbaz, Francois Schweizer, Tomasso Treu, Jason Melbourne, Dan Kelson, and Luis Ho.

The HST COSMOS Treasury program was supported through NASA grant HST-GO- 09822. We wish to thank Tony Roman, Denise Taylor, and David Soderblom for their assistance in planning and scheduling of the extensive COSMOS observations. We gratefully acknowledge the contributions of the entire COSMOS collaboration consisting of more than 70 scientists. ${ }^{15}$ It is a pleasure the acknowledge the excellent services provided by the NASA IPAC/IRSA staff(Anastasia Laity, Anastasia Alexov, Bruce Berriman and John Good) in providing online archive and server capabilities for the COSMOS data sets. We also wish to acknowledge support for the COSMOS Science meeting in May 2005 which was supported in part by NSF grant OISE-0456439.

\section{Facilities: HST(ACS)}

\footnotetext{
15 More information on the COSMOS survey is available at http://www.astro caltech.edu/ cosmos.
} 

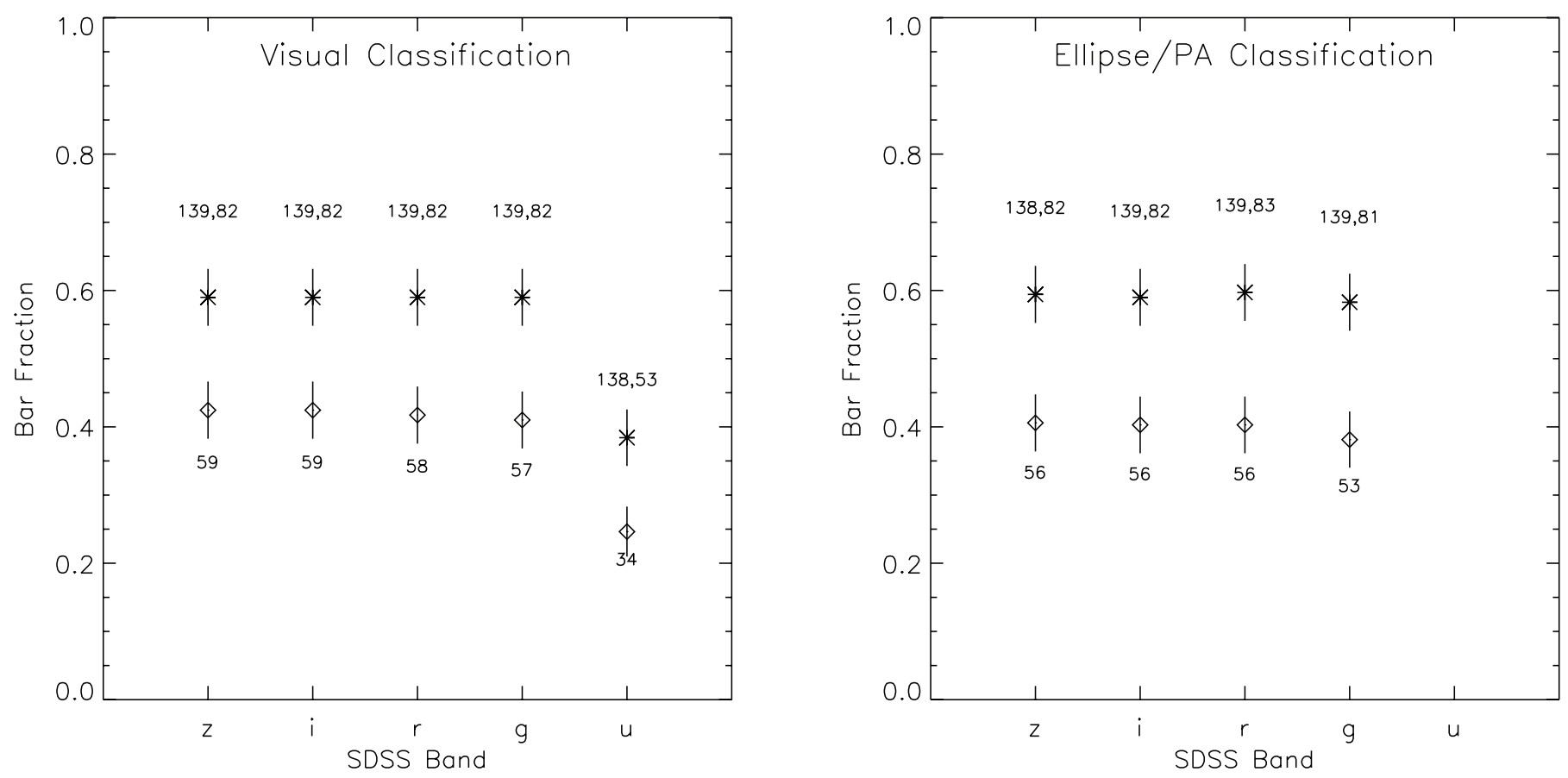

FIG. 7.-Bar fraction as a function of the SDSS filters $(u, g, r, i$, and $z)$ for a local sample of 139 SDSS galaxies. The left panel shows the results using the visual classification method and the right panel using the ellipse-fitting method described in $\S 3$. These are the same methods used for the analysis of the COSMOS data. The asterisks show the total bar fraction and the diamonds show the strong bar fraction, as described in $\S 3$. The pair of numbers above each point are the total number of galaxies and bars identified by each method. The $u$-band data point is missing in the right panel because the ellipse-fitting algorithm fails in a majority of the galaxies in the $u$ band. As noted in the text the main point of this exercise is to quantify the effects of $k$-correction on bar identification. We find that there is a significant $k$-correction for the bar fraction shortward of the Balmer break in the $u$ band but the bar fraction is constant from the $z$ band to the $g$ band.

\section{APPENDIX A}

\section{ANALYSIS OF SELECTION EFFECTS}

Although we have carefully chosen a robust sample of galaxies, used multiple methods for identifying bars and analyzed a sample of local Sloan Digital Sky Survey (SDSS) galaxies in the same manner as the COSMOS galaxies (§2), our results are in contradiction to some previous studies. Therefore we do additional investigation of the remaining possible selection effects (cosmological/surface brightness dimming and spatial resolution), which might produce a declining bar fraction.

\section{A1. $K$-CORRECTIONS AND THE EFFECTS OF BANDSHIFTING}

The ACS data for COSMOS utilizes the broad F814W filter which traces different rest-frame wavelengths at different redshifts. As a result it is imperative to understand the effects of $k$-correction (bandshifting) and correct them as necessary. To quantify the effects of $k$-correction on the identification of bars, we examined a local sample of 139 galaxies in all five Sloan bands $(u, g, r, i$, and $z)$. We selected nearby $\left(<100 \mathrm{Mpc}\right.$ ), face-on $(b / a>0.58)$, large (a $90 \%$ radius $>2 \mathrm{kpc}$ ) and bright $\left(M_{B}-19.7, M_{B}\right.$ estimated from $g-r$ colors and $g$-band magnitudes Blanton et al. 2003) spiral galaxies from the SDSS (York et al. 2000; Gunn et al. 1998) Data Release 4 (Adelman-McCarthy et al. 2006). The data were mosaicked and calibrated using the methods described by West et al. (2007).

We used the same bar identification methods (ellipse fitting and visual classification; see $\S 3$ ) for the SDSS data as for the COSMOS data and measured the bar fraction in each band. The results are shown in Figure 7. The bar fraction is unchanged from the $z$ band to the $g$ band at $f_{\text {bar }} \sim 0.6$. This is consistent with a number of previous studies (e.g., Menéndez-Delmestre et al. 2007; Eskridge et al. 2002; Whyte et al. 2002) that have shown that the overall bar fraction does not change appreciably between the optical and near-infrared bands. This figure also demonstrates that the shifting rest-wavelength of observation in our sample does not bias our measurements of the bar fraction, provided we restrict the maximum redshift of our sample appropriately.

The maximum redshift chosen is important, because Figure 7 shows that the SDSS bar fraction does appear to decline markedly in the $u$ band. At this wavelength, in a majority of cases, the ellipse-fitting technique fails completely. This is not unexpected, and a component of this decline may find its origin in the relatively poor signal-to-noise ratio of the SDSS $u$-band data. However, we suspect that the bulk of this decline is real. Bars are primarily stellar structures and some become significantly fainter and sometimes disappear altogether shortward of the Balmer break. A dramatic example of this is shown for the nearby strongly barred spiral NGC 4303 in Figure 1 of Sheth et al. (2003). This is further justification for our chosen limiting redshift in this paper, because by restricting our sample to $z=0.835$, the F814W filter does not probe bluer than rest-frame $g$ band, as shown in Figure 8 .

\section{A2. OBJECTS WITH PECULIAR MORPHOLOGY}

We considered the possibility that the bar fraction may be incorrectly measured at high redshifts due to the presence of a more exotic variety of morphologies that have been observed at high redshifts. Elmegreen et al. (2005a, 2005b, 2007; Elmegreen \& Elmegreen 


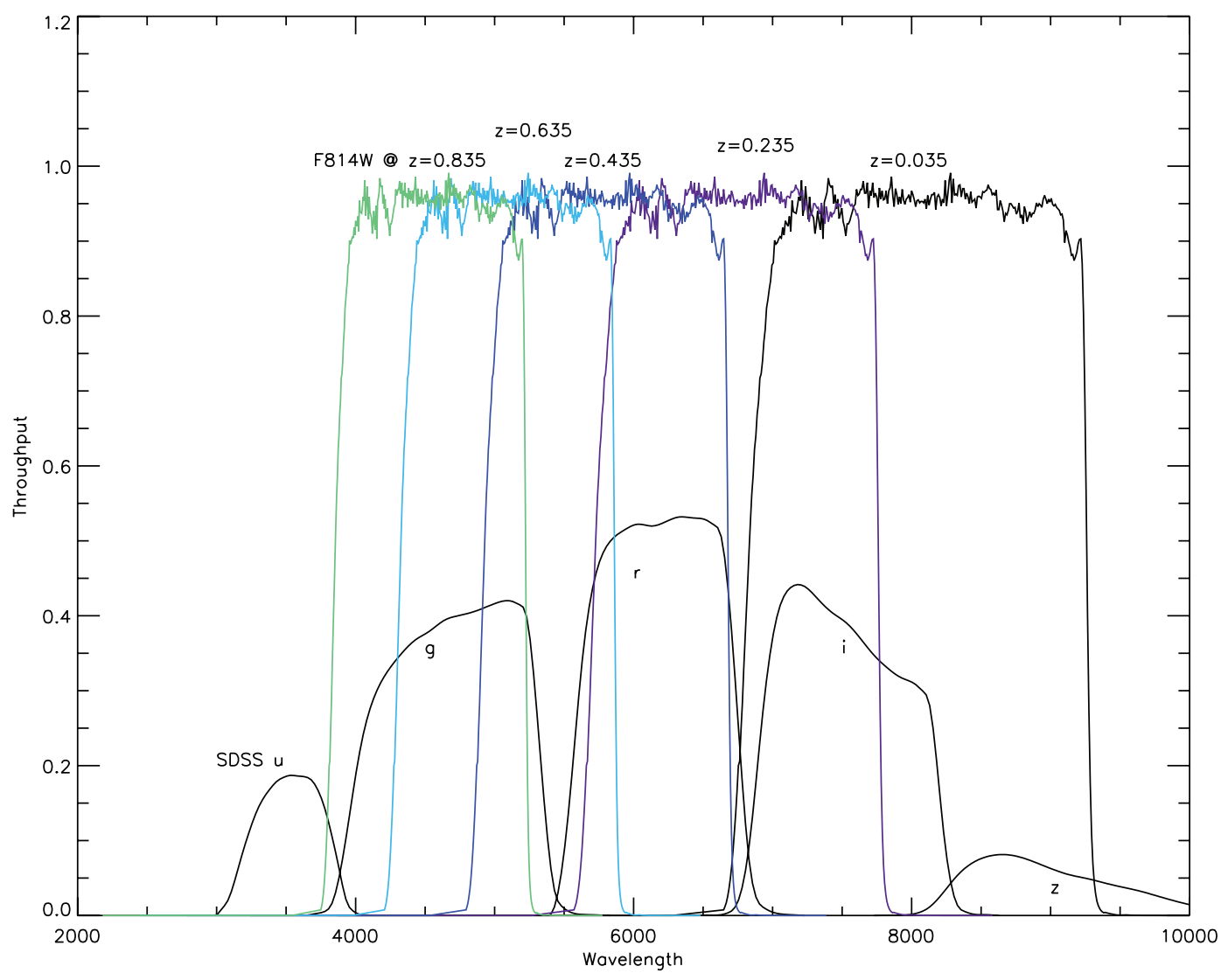

FIG. 8. - Rest-frame wavelengths traced by the F814W filter over the redshift range of this study. At the highest redshift $z=0.835$, the F814W filter is tracing the restframe SDSS $g$-band filter where the effects of $k$-correction and identification of bars is still minimally affected as discussed in Appendix A.1.

2005a, 2006) have identified clump-cluster, compact, and chain galaxies at high redshifts that do not have counterparts in the local universe. Could the $f_{\text {bar }}$ be artificially lower because we are including more of these objects in the total sample at the higher redshifts? As noted earlier, this is not the case for the visual classification method for which these objects were already excluded. We specifically exclude these objects for the ElPa classification method and recompute the evolution of the bar fraction. The results are shown in Figure 9. The drop in the bar fraction in this revised sample is less steep as expected. $f_{\text {bar }}$ drops to $0.28 \pm 0.2$ at $z=0.835$, and $f_{\mathrm{SB}}$ drops to $0.12 \pm$ 0.02 . Both methods of classifying bars thus show declines of $50 \%$ in the bar fraction from the present day to $z=0.835$.

\section{A3. COSMOLOGICAL SURFACE BRIGHTNESS DIMMING}

Surface brightness dimming is critical even at $z<1$ because it evolves so strongly with redshift $\left[\propto(1+z)^{4}\right]$. The measurement of the bar fraction may be affected if the data are too shallow because as the disk of the galaxy fades the bar, which usually has a higher mean surface brightness, may remain visible and therefore be misclassified as an inclined spiral (e.g., Jogee et al. 2002). To investigate this possibility we investigated the ability of the COSMOS data (and a few other surveys) to trace an outer disk (several times the typical bar semimajor axis) isophote as a function of redshift.

The median bar semimajor axis measured from the 2MASS Large Galaxy Atlas survey is $a_{\mathrm{bar}}=4.2 \pm 2.9 \mathrm{kpc}$ and relative size is $a_{\mathrm{bar}} / R_{25}=0.29 \pm 0.17$ (Menéndez-Delmestre et al. 2007), where $R_{25}$ is the classic $25 \mathrm{mag} \operatorname{arcsec}^{-2}$ isophote in the $B$ band. Thus the $R_{25}$ radius is at least 3 times larger than a typical bar. In the outer regions of a galaxy, $B-I=1.5$. So the corresponding limiting I-band isophote is at $\mu_{I}=23.5$. An isophote a full magnitude fainter $\left(\mu_{I}=24.5 \mathrm{mag} \mathrm{arcsec}{ }^{-2}\right)$ can therefore be safely considered to be an outer disk isophote.

In Figure 10 we show the noise-to-signal value reached for a $\mu_{I}=24.5 \mathrm{mag} \mathrm{arcsec}{ }^{-2}$ isophote fading due to cosmological surface brightness dimming. The differently colored lines are for the different SED types. It is clear from this figure that COSMOS, GOODS, $\mathrm{HDF}$, and UDF are sufficiently deep to allow one to detect the outer edges of a typical $L^{*}$ disk to $z \sim 1$. This is not the case for the shallower GEMS data, which are unable to quantify the outer disk isophotes for $L^{*}$ galaxies at $z>0.5$.

In this calculation we assume that the disks have evolved passively from $z=1$ to the present, when in fact there is a magnitude of luminosity evolution in disks from the increased star formation rate. Therefore choosing a luminosity evolution in the sample selection criteria, as we have done for the COSMOS data, further improves the signal-to-noise ratio and ability to confidently trace the outermost disk isophotes with redshift. Based on this analysis we are confident that disk dimming is not responsible for the observed decline in the bar fraction with redshift in the COSMOS data.

We further tested the effects of surface brightness dimming on the data with a second empirical check by comparing the bar fraction as a function of the observed surface brightness of the disks in each redshift bin. This test is shown in Figure 11. If indeed surface brightness dimming was responsible for a decline in the bar fraction and we were preferentially classifying lower surface brightness 


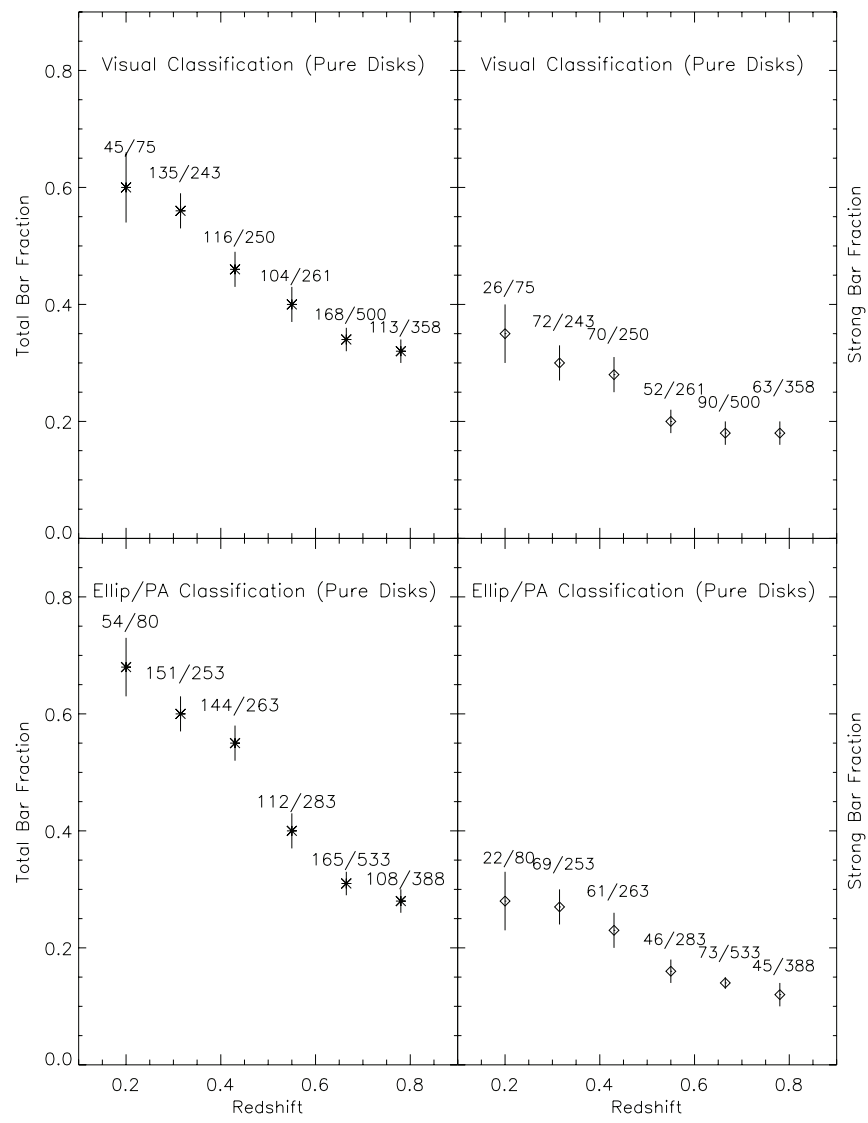

FIG. 9.- Same as for Fig. 1, except that galaxies with clump cluster, compact or other nonstandard morphologies are discarded (§ 3$)$. The overall trends noted in the discussion of Fig. 1 remain the same.

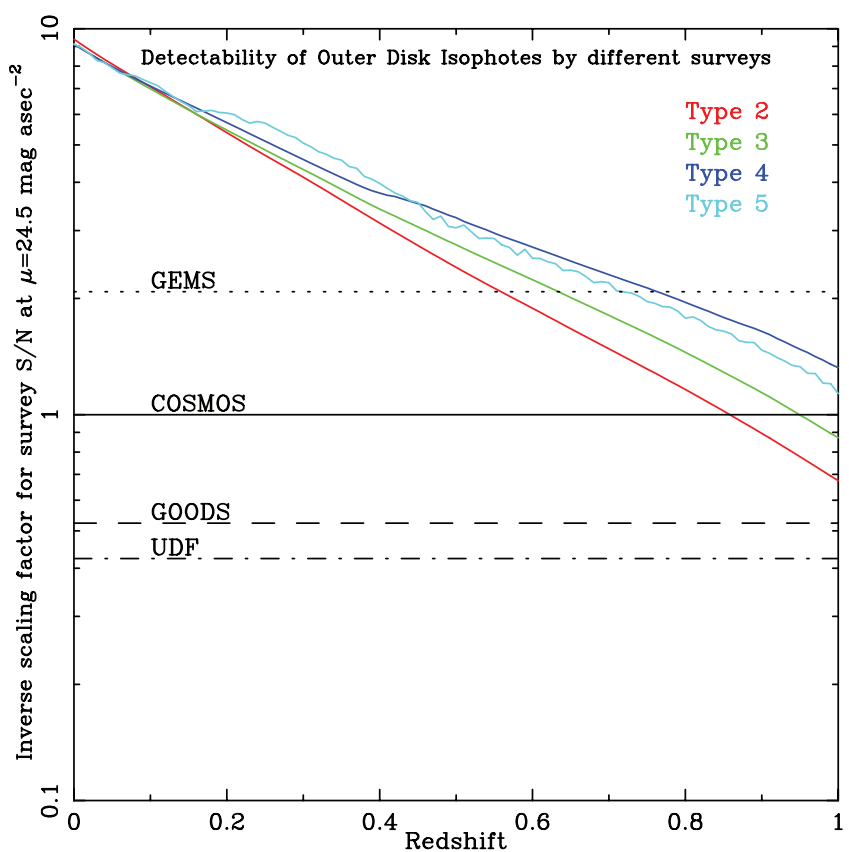

FIG. 10.-Plot of the noise-to-signal ratio for $0.6^{\prime \prime}$ diameter galaxy with a rest-frame $\mu_{I_{A} B}=24.5 \mathrm{mag} \operatorname{arcsec}^{-2}$ in COSMOS. The choice of $0.6^{\prime \prime}$ diameter was based on previous studies of galaxy sizes (Ferguson et al. 2004). Our own data are consistent with these size estimates. We find that the median exponential scale length of $L^{*}$ galaxies in COSMOS is $3.1 \mathrm{kpc}\left(0.39^{\prime \prime}\right)$ at $z=0.835$ (Sheth et al. 2008). In the sample analyzed here, $93 \%$ of the galaxies have a half-light radius greater than $0.3^{\prime \prime}$. So the noise to signal measurement shown here for COSMOS data for the outermost disk isophote is a lower limit. The horizontal lines show the $1 \sigma$ sensitivity limits for the COSMOS, GEMS, GOODS, and UDF survey. Note that GEMS will be adversely affected in measurements $f_{\text {bar }}$ even for $L^{*}$ galaxies at $z>0.5$ because of the low signal-tonoise ratio in the underlying disk. 


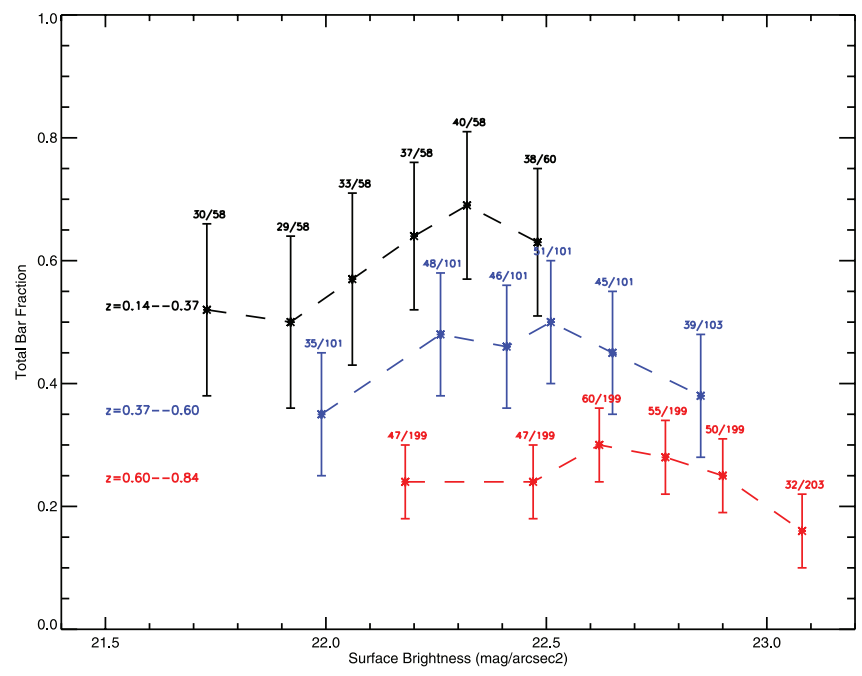

Fig. 11.-Plot of the total bar fraction $\left(f_{\text {bar }}\right)$ vs. measured surface brightness in the COSMOS sample in three redshift bins. We find that there is no correlation between the bar fraction and the surface brightness of the galaxies, as might have been expected if surface brightness dimming was affecting the measurement of the bar fraction. The overall trend of lower bar fraction with redshift can be seen readily in these panels. The results are the same when considering only the strong bar fraction.

disks as unbarred, we should see a correlation of $f_{\text {bar }}$ with $\mu$. We see no trends in the bar fraction with $\mu$ in any redshift bin. Thus we conclude that the observed evolution of the bar fraction shown in Figure 1 is not due to cosmological surface brightness dimming of the disks.

\section{A4. SPATIAL RESOLUTION AND THE BAR FRACTION}

One possibility for the observed decline in the bar fraction could be that we are preferentially missing small bars at higher redshifts. As already noted earlier (and as shown in Fig. 3 of Sheth et al. 2003), CS resolution (1 pixel $=0.05^{\prime \prime}=0.38 \mathrm{kpc}$ at $z=0.835$ ) is adequate for identifying all bars larger than $2 \mathrm{kpc}$ at all $z<0.835$. Bars smaller than this, at least in the local universe, are nuclear bars and not the primary bars that we are concerned with in this paper. Very small bars in late-type galaxies have been measured by Erwin (2005) in a local sample, but it is unclear whether these galaxies are comparable to the large, $L^{*}$ and brighter galaxies being examined in our sample. For $L^{*}$ galaxies, there is substantial evidence that the size of galaxy disks does not change significantly to $z \sim 1$. For the COSMOS sample, the median disk scale length is unchanged over the redshift range under investigation, consistent with similar results found previously (Ravindranath et al. 2004; Barden et al. 2005; Sargent et al. 2007). Given that the bar semimajor axis is typically $0.3 R_{25}$, the lack of a change in disk sizes also indicates that it is unlikely that bars change their sizes significantly as a function of redshift.

As another check we decided to see if the measurement of the bar fraction was affected by the size of galaxies in a given redshift bin. If the bar fraction was indeed declining because of smaller galaxy disks with unresolved bars, one would expect to see a decreasing $f_{\text {bar }}$ with smaller disks and this effect would be most pronounced at higher redshifts where the linear resolution of the ACS data is the coarsest. When we plot $f_{\text {bar }}$ versus the half light radius of galaxies in different redshift bins in Figure 12, we find no significant correlation between the bar fraction and the size of the disk in any of the redshift bins. There is a slight decline in the bar fraction for the smallest galaxies. This reflects the finding that bars are less frequent in less massive systems and not a selection effect. The result is the same when considering only $f_{\mathrm{SB}}$, or using the exponential scale length for an estimate of the galaxy disk size. We therefore conclude that there is not a preferential loss of bars in disks of any particular size at any redshift in this study.

\section{APPENDIX B}

\section{ARTIFICIALLY REDSHIFTING GALAXIES}

The above sections have addressed most of the classical selection effects that plague high-redshift studies, namely, $k$-correction, resolution, surface brightness dimming, etc. To conclusively test all of these effects, we artificially redshifted the $g$-band images of all 139 SDSS galaxies to $z=0.84$ following the technique outlined in Giavalisco et al. (1996). We redshifted the galaxies to the F814W filter, rebinned the image, took into account surface brightness dimming and matched the noise characteristics of our ACS data. Examples of the artificially redshifted galaxies are shown in Figure 13. We reclassified these images into strongly barred, weakly barred and unbarred spirals as we had done before. In 127/139 galaxies the classification remained unchanged. Of the remaining twelve galaxies, seven are now classified as weakly barred in these images compared to the original SDSS images where they were classified as unbarred. Four galaxies classified previously as weakly barred are now classified as unbarred. And one galaxy that was classified as strongly barred is now classified as an unbarred spiral. So overall the bar fraction did not change appreciably (original SDSS images $f_{\text {bar }}=0.59$, artificially redshifted SDSS images $f_{\text {bar }}=0.60$ ). These results are remarkable but not unexpected and reflect the exquisite sensitivity of the ACS survey, which was designed to be as deep at $z=1$ as the SDSS survey is locally. 


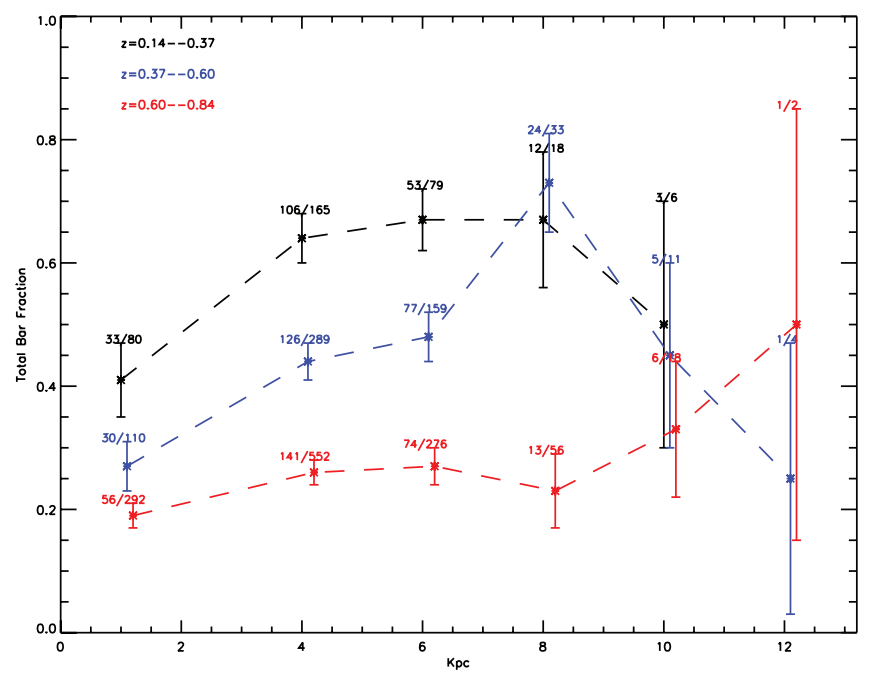

FIG. 12.-Plot of the total bar fraction $\left(f_{\text {bar }}\right)$ vs. half-light radius. If the bar fraction was indeed declining because of smaller galaxy disks with unresolved bars, one would expect to see a decreasing $f_{\text {bar }}$ with smaller disks particularly in the highest redshift bin. We find no such trend and therefore conclude that the general decline in the bar fraction is not correlated with disk size.
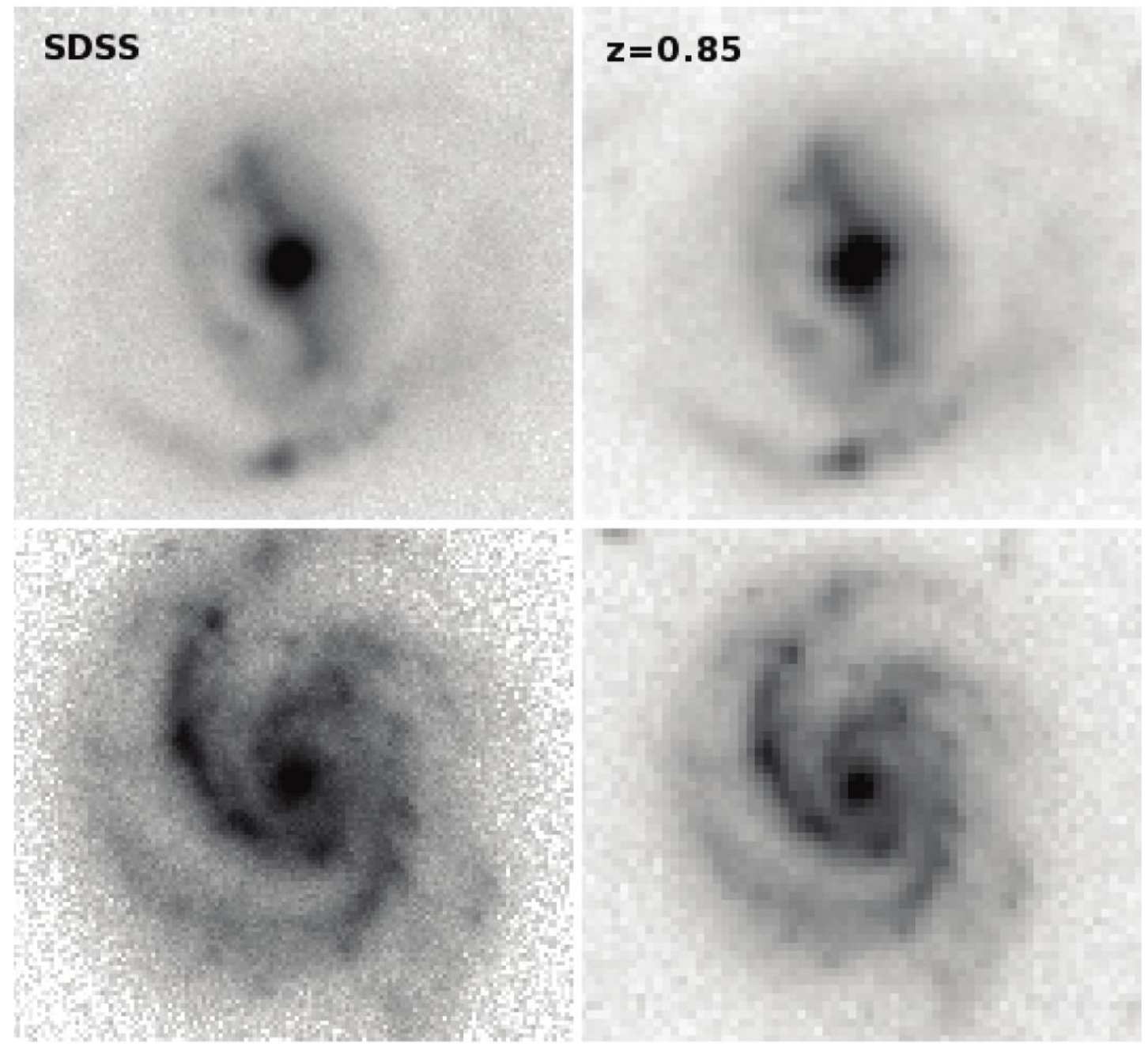

Fig. 13.- Two examples of artificially redshifted SDSS galaxies. A barred spiral is shown in the top row and a spiral galaxy in the bottom row. The left column shows the original SDSS $g$-band image and the right column is the artificially redshifted image at $z=0.84$ 
Abraham, R. G., Merrifield, M. R., Ellis, R. S., Tanvir, N. R., \& Brinchmann, J. 1999, MNRAS, 308, 569

Abraham, R. G., Tanvir, N. R., Santiago, B. X., Ellis, R. S., Glazebrook, K., \& van den Bergh, S. 1996, MNRAS, 279, L47

Adelman-McCarthy, J. K., et al. 2006, ApJS, 162, 38

Arnouts, S., et al., ApJ, 2005, 619, L43

Athanassoula, E. 2002, ApJ, 569, L83

$$
\text { 2003a, MNRAS, 341, } 1179
$$

- 2005, MNRAS, 358, 1477

Athanassoula, E., Lambert, J. C., \& Dehnen, W. 2005, MNRAS, 363, 496

Athanassoula, E., \& Sellwood, J. A. 1986, MNRAS, 221, 213

Barazza, F. B., Jogee, S., \& Marinova, I. 2008, ApJ, in press (arXiv: 0710.4674)

Barden, M., et al. 2005, ApJ, 635, 959

Bell, E. F., et al. 2005, ApJ, 625, 23

Berentzen, I., Athanassoula, E., Heller, C., \& Fricke, K. 2004, MNRAS, 347, 220

Berentzen, I., Heller, C. H., Shlosman, I., \& Fricke, K. J. 1998, MNRAS, 300, 49

Blanton, M. R., et al. 2003, AJ, 125, 2348

Block, D. L., Bournaud, F., Combes, F., Puerari, I., \& Buta, R. 2002, A\&A, 394, L35

Bosma, A. 2004, in IAU Symp. 220, Dark Matter in Galaxies, ed. S. D. Ryder et al. (San Francisco: ASP), 39

Bournaud, F., \& Combes, F. 2002, A\&A, 392, 83

Bournaud, F., \& Combes, F., \& Semelin, B. 2005, MNRAS, 364, L18

Bundy, K., et al. 2006, ApJ, 651, 120

Bunker, A. J. 1999, in ASP Conf. Ser. 191, Photometric Redshifts and the Detection of High Redshift Galaxies, ed. R. Weymann et al. (San Francisco: ASP) 317

Buta, R., Laurikainen, E., \& Salo, H. 2004, AJ, 127, 279

Buta, R., Vasylyev, S. Salo, H., \& Laurikainen, E. 2005, AJ, 130, 506

Capak, P. 2003, Ph.D. thesis, Univ. Hawaii

Capak, P., et al. 2007, ApJS, 172, 284

Coleman, G. D., Wu, C.-C., \& Weedman, D. W. 1980, ApJS, 43, 393

Cowie, L. L., Songaila, A., Hu, E. M., \& Cohen, J. G. 1996, AJ, 112, 839

Daddi, E., et al. 2004, ApJ, 600, L127 2005, ApJ, 631, L13

Das, M., Vogel, S. N., Teuben, P. J., Regan, M. W., Sheth, K., Harris, A. I., \& Jeffreys, W. H. 2003, ApJ, 582, 190

de Vaucouleurs, G., de Vaucouleurs, A., Corwin, H. G., Buta, Jr., R. J., Paturel, G., \& Fouqué, P. 1991, Third Reference Catalogue of Bright Galaxies (New York: Springer) ( RC3)

Elmegreen, D. M., \& Elmegreen, B. G. 1982, MNRAS, 201, 1021

- 2005, ApJ, 627, 632

2006, ApJ, 651, 676

Elmegreen, B. G., Elmegreen, D. M., \& Hirst, A. C. 2004, ApJ, 612, 191

Elmegreen, D. M., Elmegreen, B. G., Ravindranath, S., \& Coe, D. A. 2007, ApJ, 658, 763

Elmegreen, D. M., Elmegreen, B. G., Rubin, D. S., \& Schaffer, M. A. 2005a, ApJ, 631, 85

Elmegreen, B. G., Elmegreen, D. M., Vollbach, D. R., Foster, E. R., \& Ferguson, T. E. 2005b, ApJ, 634, 101

Erwin, P. 2005, MNRAS, 364, 283

Eskridge, P., et al. 2000, AJ, 119, 536

- 2002, ApJS, 143, 73

Ferrarese, L., \& Merritt, D. 2000, ApJ, 539, L9

Ferguson, H. C., et al. 2004, ApJ, 600, L107

Fontana, A., et al. 2004, A\&A, 424, 23

Friedli, D., \& Benz, W. 1993, A\&A, 268, 65

Fukuda, H., Habe, A., \& Wada, K. 2000, ApJ, 529, 109

Gerin, M., Combes, F., \& Athanassoula, E. 1990, A\&A, 230, 37

Giavalisco, M., Livio, M., Bohlin, R. C., Macchetto, F. D., \& Stecher, T. P. 1996, AJ, 112, 369

Glazebrook, K., et al. 2004, Nature, 430, 181
Gunn, J. E., et al. 1998, AJ, 116, 3040

Ilbert, O., et al. 2006, A\&A, 453, 809

Jogee, S., Kenney, J.D.P., \& Smith, B. J. 1999, ApJ, 526, 665

Jogee, S., Knapen, J. H., Laine,S., Shlosman, I., \& Scoville, N. Z. 2002, ApJ, 570 , L55

Jogee, S., et al. 2004, ApJ, 615, L105

Kartaltepe, J. S., et al. 2007, ApJS, 172, 320

Kassin, S. A., et al. 2007, ApJ, 660, L35

Kinney, A. L., Calzetti, D., Bohlin, R. C., McQuade, K., Storchi-Bergmann, T., \& Schmitt, H. R. 1996, ApJ, 467, 38

Knapen, J. H., Shlosman, I., \& Peletier, R. F. 2000, ApJ, 529, 93

Kormendy, J., \& Kennicutt, R. C., Jr. 2004, ARA\&A, 42, 603

Kormendy, J., Norman, 1979, ApJ, 233, 539

Kormendy, J., \& Richstone, D. 1995, ARA\&A, 33, 581

Kranz, T., Slyz, A., \& Rix, H.-W. 2003, ApJ, 586, 143

Laine, S., Shlosman, I., Knapen, J. H., \& Peletier, R. F. 2002, ApJ, 567, 97

Magorrian, J., et al. 1998, AJ, 115, 2285

Maier, C., Lilly, S. J., Carollo, C. M., Meisenheimer, K., Hippelein, H., \& Stockton, A. 2006, ApJ, 639, 858

Maier, C., Lilly, S. J., Carollo, C. M., Stockton, A., \& Brodwin, M. 2005, ApJ, 634, 849

Maraston, C. 2005, MNRAS, 362, 799

Marinova, I., \& Jogee, S. 2007, ApJ, 659, 1176

Menéndez-Delmestre, K., Sheth, K., Schinnerer, E., Jarrett, T. H., \& Scoville, N. Z. 2007, ApJ, 657, 790

Mobasher, B., et al. 2007, ApJS, 172, 117

Nilson, P. 1973, Uppsala General Catalogue of Galaxies (Uppsala: Astron. Obs.)

Noguchi, M. 1987, MNRAS, 228, 635

Pei, Y. C. 1995, ApJ, 438, 623

Peng, C. Y., Ho, L. C., Impey, C. D., \& Rix, H.-W. 2002, AJ, 124, 266

Ravindranath, S., et al. 2004, ApJ, 604, L9

Regan, M. W., \& Elmegreen, D. M. 1997, AJ, 114, 965

Sakamoto, K., Okumura, S. K., Ishizuki, S., \& Scoville, N. Z. 1999b, ApJ, 525, 691

Sandage, A., \& Tammann, G. A. 1987, A Revised Shapley-Ames Catalog of Bright Galaxies (2nd. ed.; Washington: Carnegie Inst. Washington)

Sargent, M. T., et al. 2007, ApJS, 172, 434

Scarlata, C., et al. 2007a, ApJS, 172, 406 2007b, ApJS, 172, 494

Scoville, N. Z., et al. 2007a, ApJS, 172, 1

2007b, ApJS, 172, 38

Shen, J., \& Sellwood, J. A. 2004, ApJ, 604, 614

Sheth, K., Regan, M. W., Scoville, N. Z., \& Strubbe, L. E. 2003, ApJ, 592, L13

Sheth, K., Regan, M. W., Vogel, S. N., \& Teuben, P. J. 2000, ApJ, 532, 221

Sheth, K., Vogel, S. N., Regan, M. W., Teuben, P. J., Harris, A. I., \& Thornley, M. D. 2002, AJ, 124, 2581

Sheth, K., Vogel, S. N., Regan, M. W., Teuben, P. J., Harris, A. I., Thornley, M. D., \& Helfer, T. T. 2005, ApJ, 632, 217

Sheth, K., et al. 2008, in ASP Conf. Ser., Pathways through an Eclectic Universe, ed. J. H. Knapen, T. J. Mahoney, \& A. Vazdekis (San Francisco: ASP), in press Steinmetz, M., \& Navarro, J. F. 2002, NewA, 7, 155

Toomre, A., \& Toomre, J. 1972, ApJ, 178, 623

Treu, T., Ellis, R. S., Liao, T. X., \& van Dokkum, P. G. 2005, ApJ, 622, L5

Ueda, Y., Akiyama, M., Ohta, K., \& Miyaji, T. 2003, ApJ, 598, 886

van den Bergh, S., Abraham, R. G., Ellis, R. S., Tanvir, N. R., Santiago, B., \& Glazebrook, K. G. 1996, AJ, 112, 359

West, A. A., Garcia-Appadoo, D. A., Dalcanton, J. J., Disney, M. J., Rockosi, C. M., Ivezic, Z., \& Bentz, M. C. 2007, AJ, submitted

Whyte, L. F., Abraham, R. G., Merrifield, M. R., Eskridge, P. B., Frogel, J. A., \& Pogge, R. W. 2002, MNRAS, 336, 1281

York, D. G., et al. 2000, AJ, 120, 1579 